\title{
UNIFORM RECTIFIABILITY FROM CARLESON MEASURE ESTIMATES AND $\varepsilon$-APPROXIMABILITY OF BOUNDED HARMONIC FUNCTIONS
}

\author{
JOHN GARNETT, MIHALIS MOURGOGLOU, AND XAVIER TOLSA
}

\begin{abstract}
Let $\Omega \subset \mathbb{R}^{n+1}, n \geq 1$, be a corkscrew domain with Ahlfors-David regular boundary. In this paper we prove that $\partial \Omega$ is uniformly $n$-rectifiable if every bounded harmonic function on $\Omega$ is $\varepsilon$-approximable or if every bounded harmonic function on $\Omega$ satisfies a suitable square-function Carleson measure estimate. In particular, this applies to the case when $\Omega=\mathbb{R}^{n+1} \backslash E$ and $E$ is Ahlfors-David regular. Our results solve a conjecture posed by Hofmann, Martell, and Mayboroda in a recent work where they proved the converse statements. Here we also obtain two additional criteria for uniform rectifiability. One is given in terms of the so-called " $S<N$ " estimates, and another in terms of a suitable corona decomposition involving harmonic measure.
\end{abstract}

\section{INTRODUCTION}

In this paper we characterize the uniform $n$-rectifiability of the boundary of a domain $\Omega \subset \mathbb{R}^{n+1}, n \geq 1$, in terms of a square function Carleson measure estimate, or of an approximation property, for the bounded harmonic functions on $\Omega$. Our results solve an open problem posed by Hofmann, Martell and Mayboroda in [HMM2].

We introduce some definitions and notations. A set $E \subset \mathbb{R}^{d}$ is called $n$-rectifiable if there are Lipschitz maps $f_{i}: \mathbb{R}^{n} \rightarrow \mathbb{R}^{d}, i=1,2, \ldots$, such that

$$
\mathcal{H}^{n}\left(E \backslash \bigcup_{i} f_{i}\left(\mathbb{R}^{n}\right)\right)=0
$$

where $\mathcal{H}^{n}$ stands for the $n$-dimensional Hausdorff measure. A set $E \subset \mathbb{R}^{d}$ is called $n$-ADregular (or just AD-regular or Ahlfors-David regular) if there exists some constant $c>0$ such that

$$
c_{0}^{-1} r^{n} \leq \mathcal{H}^{n}(B(x, r) \cap E) \leq c_{0} r^{n} \quad \text { for all } x \in E \text { and } 0<r \leq \operatorname{diam}(E) .
$$

The set $E \subset \mathbb{R}^{d}$ is uniformly n-rectifiable if it is $n$-AD-regular and there exist constants $\theta, M>0$ such that for all $x \in E$ and all $0<r \leq \operatorname{diam}(E)$ there is a Lipschitz mapping $g$ from the ball $B_{n}(0, r)$ in $\mathbb{R}^{n}$ to $\mathbb{R}^{d}$ with $\operatorname{Lip}(g) \leq M$ such that

$$
\mathcal{H}^{n}\left(E \cap B(x, r) \cap g\left(B_{n}(0, r)\right)\right) \geq \theta r^{n} .
$$

The analogous notions for measures are the following. A Radon measure $\mu$ on $\mathbb{R}^{d}$ is $n$ rectifiable if it vanishes outside an $n$-rectifiable set $E \subset \mathbb{R}^{d}$ and if moreover $\mu$ is absolutely

2010 Mathematics Subject Classification. 31A15, 28A75, 28 A78.

J.G. was supported by NSF Grant DMS 1217239. M.M was supported by the ERC grant 320501 of the European Research Council (FP7/2007-2013) and also by the Basque Government through the BERC 20142017 program and by Spanish Ministry of Economy and Competitiveness MINECO: BCAM Severo Ochoa excellence accreditation SEV-2013-0323. X.T. was supported by the ERC grant 320501 of t7/2007-2013) and also by 2014-SGR-75 (Catalonia), MTM2013-44304-P (Spain), and the Marie Curie ITN MAnET (FP7607647). 
continuous with respect to $\left.\mathcal{H}^{n}\right|_{E}$. On the other hand, $\mu$ is called $n$-AD-regular if it is of the form $\mu=\left.g \mathcal{H}^{n}\right|_{E}$, where $E$ is $n$-AD-regular and $g: E \rightarrow(0,+\infty)$ satisfies $g(x) \approx 1$ for all $x \in E$, with the implicit constant independent of $x$. If, moreover, $E$ is uniformly $n$-rectifiable, then $\mu$ is called uniformly n-rectifiable.

The notion of uniform rectifiability should be considered a quantitative version of rectifiability. It was introduced in their pioneering works [DS1], [DS2] of David and Semmes, who were seeking a good geometric framework under which all singular integrals with odd and sufficiently smooth kernel are bounded in $L^{2}$.

An open set $\Omega \subset \mathbb{R}^{n+1}$ is called a corkscrew domain if for every ball $B(x, r)$ with $x \in \partial \Omega$ and $0<r \leq \operatorname{diam}(\Omega)$ there exists another ball $B\left(x^{\prime}, r^{\prime}\right) \subset \Omega \cap B(x, r)$ with radius $r^{\prime} \approx r$, with the implicit constant independent of $x$ and $r$. Let us remark that we do not ask $\Omega$ to be connected. For example, if $E \subset \mathbb{R}^{n+1}$ is a closed $n$-AD-regular set, then it follows easily that $\mathbb{R}^{n+1} \backslash E$ is a corkscrew domain.

Let $\Omega \subset \mathbb{R}^{n+1}$ be open, and let $u$ be a bounded harmonic function on $\Omega$. For $\varepsilon>0$ we say that $u$ is $\varepsilon$-approximable if there is $\varphi \in W_{\mathrm{loc}}^{1,1}(\Omega)$ and $C>0$ such that

$$
\|u-\varphi\|_{L^{\infty}(\Omega)}<\varepsilon
$$

and for all $x \in \partial \Omega$ and all $r>0$

$$
\frac{1}{r^{n}} \int_{B(x, r)}|\nabla \varphi(y)| d y \leq C,
$$

where $d y$ denotes the Lebesgue measure in $\mathbb{R}^{n+1}$. It is clear by a normal family argument that every bounded harmonic function on $\Omega$ is $\varepsilon$-approximable for all $\varepsilon>0$ if and only if (1.2) and (1.3) hold for all harmonic $u$ with $\|u\|_{L^{\infty}(\Omega)} \leq 1$ with constant $C=C_{\varepsilon}$ depending on $\varepsilon$ but not on $u$. The notion of $\varepsilon$-approximability was introduced by Varopoulos in [Va] in connection with corona problems. See [Gar, Chapter VIII] for a proof on the upper half plane and [Dah], for the case of Lipschitz domains. Also see [Gar], [HMM2], [KKiPT], [KKoPT], and [Pi] for further results and applications, including some to elliptic operators.

Our main result is the following:

Theorem 1.1. Let $\Omega \subset \mathbb{R}^{n+1}, n \geq 1$, be a corkscrew domain with $n$-AD-regular boundary. Then the following are equivalent:

(a) $\partial \Omega$ is uniformly n-rectifiable.

(b) Every bounded harmonic function on $\Omega$ is $\varepsilon$-approximable for all $\varepsilon>0$.

(c) There is $C>0$ such that if $u$ is a bounded harmonic function on $\Omega$ and $B$ is a ball centered at $\partial \Omega$,

$$
\int_{B}|\nabla u(x)|^{2} \operatorname{dist}(x, \partial \Omega) d x \leq C\|u\|_{L^{\infty}(\Omega)}^{2} r(B)^{n} .
$$

The implications (a) $\Rightarrow$ (b) and (a) $\Rightarrow$ (c) have already been proved by Hofmann, Martell, and Mayboroda in [HMM2] for $n \geq 2$, but a careful reading of their proof shows same implications hold for $n=1$ with small modifications. In the current paper we will only prove that (b) $\Rightarrow$ (a) and that (c) $\Rightarrow$ (a), but in a slightly stronger formulation because we only assume (b) or (c) holds for bounded functions continuous on $\bar{\Omega}$ and harmonic on $\Omega$.

As a corollary of the preceding theorem we deduce another characterization of uniform rectifiability in terms of a square function - nontangential maximal function estimate (of the 
type " $S<N$ ") in the case $n \geq 2$. To state this result we need some additional notation. Given $x \in \partial \Omega$, we define the cone

$$
\Gamma(x)=\{y \in \Omega:|x-y|<2 \operatorname{dist}(y, \partial \Omega)\}
$$

and for a continuous function $u$ in $\Omega$, we define the non-tangential maximal function

$$
N_{*} u(x)=\sup _{y \in \Gamma(x)}|u(y)| .
$$

For $u \in W_{l o c}^{1,2}(\Omega)$ we also define the square function

$$
S u(x)=\left(\int_{y \in \Gamma(x)}|\nabla u(y)|^{2} \operatorname{dist}(y, \partial \Omega)^{1-n} d y\right)^{1 / 2} .
$$

Then we have:

Corollary 1.2. Let $\Omega \subset \mathbb{R}^{n+1}, n \geq 2$, be a corkscrew domain with $n$-AD-regular boundary. Denote by $\mu$ the surface measure on $\partial \Omega$. Suppose that for some $p \in[2, \infty)$ there exists some constant $C_{p}>0$ such that for every function $u \in C_{0}(\bar{\Omega})$ harmonic in $\Omega$,

$$
\|S u\|_{L^{p}(\mu)} \leq C_{p}\left\|N_{*} u\right\|_{L^{p}(\mu)}
$$

Then $\partial \Omega$ is uniformly rectifiable.

In Hofmann and Le [HL, eq. (4.12)] the estimate (1.5) (at least for $n \geq 2$ ) is asserted for corkscrew domains with uniformly $n$-rectifiable boundaries and attributed to a forthcoming paper by Hofmann, Martell and Mayboroda. From that paper and Corollary 1.2 it follows that (1.5) also characterizes uniform rectifiability for corkscrew domain with $n$-AD-regular boundary for $n \geq 2$.

We will prove Theorem 1.1 by using the connection between harmonic measure the Riesz transforms and then applying the result from [NToV1] that the $L^{2}(\mu)$ boundedness of the vector or Riesz transforms implies the uniform $n$-rectfiability of $\mu$. The connection between harmonic measure and uniform $n$-rectifiability has been a subject of intensive research in the last years. See for example, [DJ], [HM1], [HMU], [HMM1], [AHMNT], [BH], and [HM2]. Among these we would like to highlight [HM1] and [HMU], from which it follows that for a bounded uniform domain $\Omega \subset \mathbb{R}^{n+1}$ (so that $\partial \Omega$ is $n$-AD-regular), the harmonic measure $\omega^{p}$ in $\Omega$ is an $A_{\infty}$ weight with respect to the surface measure if and only if $\partial \Omega$ is uniformly $n$-rectifiable. On the other hand, the connection between harmonic measure and the Riesz transforms, in combination with the rectifiability criteria from [NToV1] and [NToV2], has been successfully exploited in other recent works such as [AHM $\left.{ }^{3} \mathrm{TV}\right],[\mathrm{MT}]$, and [AMT2].

To show that the Riesz transform vector is bounded in $L^{2}(\mu)$ we will use a corona type decomposition. Unlike the usual corona decompositions of David and Semmes in [DS2], which are of geometric nature, the one we will need is based on the comparison between surface measure and harmonic measure. We will derive the usual packing condition for such decomposition from the assumptions (b) or (c) in Theorem 1.1 with a suitable test function $u$ whose construction involves the harmonic measure. Then, by a comparison argument between surface measure and harmonic measure we will prove the boundedness of the Riesz transform "at the scale of each tree" of the corona decomposition. To implement this argument is a non-trivial task as, for example, both measures may be mutually singular. To overcome these technical difficulties we will use the suppressed Riesz kernels introduced 
by Nazarov, Treil and Volberg in $[\mathrm{NTrV}]$ and the sophisticated $T b$ theorems they proved for such operators.

Finally, we remark that the corona decomposition we use in the proof of Theorem 1.1 provides a new characterization, in terms of harmonic measure, of uniform rectifiability for boundaries of corkscrew domains. This complements, in some sense, another characterization in terms of big pieces of NTA domains, recently obtained by Bortz and Hofmann [BH] and Martell and Hofmann [HM2]. This corona decomposition characterization is described in Propositions 3.1 and 5.1, but an equivalent and somewhat less technical form of it is provided by the following theorem.

Theorem 1.3. Let $\Omega \subset \mathbb{R}^{n+1}$ be a corkscrew domain with $n$-AD-regular boundary. Denote by $\mu$ the surface measure on $\partial \Omega$, and let $\mathcal{D}_{\mu}$ be a dyadic lattice of cubes associated to $\mu$ as in Subsection 2.1. Then $\partial \Omega$ is uniformly $n$-rectifiable if and only if there exists a family $\mathcal{F} \subset \mathcal{D}_{\mu}$ satisfying the following properties:

(a) Every cube $Q \in \mathcal{D}_{\mu}$ is contained in some cube $R \in \mathcal{F}$.

(b) The family $\mathcal{F}$ fulfills the packing condition

$$
\sum_{R \subset S: R \in \mathcal{F}} \mu(R) \leq C \mu(S) \quad \text { for all } S \in \mathcal{D}_{\mu} .
$$

(c) For each $R \in \mathcal{F}$ there exists a corkscrew point $p_{R} \in \Omega$ with

$$
c^{-1} \ell(R) \leq \operatorname{dist}\left(p_{R}, R\right) \leq \operatorname{dist}\left(p_{R}, \partial \Omega\right) \leq c \ell(R)
$$

such that, if $R$ is the smallest cube from $\mathcal{F}$ containing some cube $Q \in \mathcal{D}_{\mu}$, then

$$
\omega^{p_{R}}(5 Q) \approx \frac{\mu(Q)}{\mu(R)}
$$

with the implicit constant uniform on $Q$ and $R$.

\section{PRELiminaries}

As usual in harmonic analysis, we denote by $C$ or $c$ constants which usually only depend on the dimension $n$ and other fixed parameters (such as the constants involved in the ADregularity of $\partial \Omega$ or the corkscrew condition of $\Omega$ ), and which may change their values at different occurrences. On the contrary, constants with subscripts such as $c_{0}$ or $C_{0}$, do not change their values. For $a, b \geq 0$, we will write $a \lesssim b$ if there is $C>0$ so that $a \leq C b$ and $a \lesssim t b$ if the constant $C$ depends on the parameter $t$. We write $a \approx b$ to mean $a \lesssim b \lesssim a$ and define $a \approx_{t} b$ similarly.

2.1. Dyadic lattices. Given an $n$-AD-regular measure $\mu$ in $\mathbb{R}^{n+1}$ we consider the dyadic lattice of "cubes" built by David and Semmes in [DS2, Chapter 3 of Part I]. These dyadic cubes are not true cubes, but they play the role of cubes with respect to a given $n$-AD-regular measure $\mu$. The properties satisfied by $\mathcal{D}_{\mu}$ are the following. Assume first, for simplicity, that $\operatorname{diam}(\operatorname{supp} \mu)=\infty$. Then for each $j \in \mathbb{Z}$ there exists a family $\mathcal{D}_{\mu, j}$ of Borel subsets of $\operatorname{supp} \mu$ (the dyadic cubes of the $j$-th generation) such that:

(a) each $\mathcal{D}_{\mu, j}$ is a partition of $\operatorname{supp} \mu$, i.e. $\operatorname{supp} \mu=\bigcup_{Q \in \mathcal{D}_{\mu, j}} Q$ and $Q \cap Q^{\prime}=\varnothing$ whenever $Q, Q^{\prime} \in \mathcal{D}_{\mu, j}$ and $Q \neq Q^{\prime}$; 
(b) if $Q \in \mathcal{D}_{\mu, j}$ and $Q^{\prime} \in \mathcal{D}_{\mu, k}$ with $k \leq j$, then either $Q \subset Q^{\prime}$ or $Q \cap Q^{\prime}=\varnothing$;

(c) for all $j \in \mathbb{Z}$ and $Q \in \mathcal{D}_{\mu, j}$, we have $2^{-j} \lesssim \operatorname{diam}(Q) \leq 2^{-j}$ and $\mu(Q) \approx 2^{-j n}$;

(d) there exists $C>0$ such that, for all $j \in \mathbb{Z}, Q \in \mathcal{D}_{\mu, j}$, and $0<\tau<1$,

$$
\begin{aligned}
\mu(\{x \in Q: & \left.\left.\operatorname{dist}(x, \operatorname{supp} \mu \backslash Q) \leq \tau 2^{-j}\right\}\right) \\
& +\mu\left(\left\{x \in \operatorname{supp} \mu \backslash Q: \operatorname{dist}(x, Q) \leq \tau 2^{-j}\right\}\right) \leq C \tau^{1 / C} 2^{-j n} .
\end{aligned}
$$

Property (d) is often called the small boundaries condition. From (2.1), it follows that there is a point $z_{Q} \in Q$ (the center of $Q$ ) such that $\operatorname{dist}\left(z_{Q}, \operatorname{supp} \mu \backslash Q\right) \gtrsim 2^{-j}$ (see [DS2, Lemma 3.5 of Part I]). We set $\mathcal{D}_{\mu}:=\bigcup_{j \in \mathbb{Z}} \mathcal{D}_{\mu, j}$.

In the case $\operatorname{diam}(\operatorname{supp} \mu)<\infty$, the families $\mathcal{D}_{\mu, j}$ are only defined for $j \geq j_{0}$, with $2^{-j_{0}} \approx \operatorname{diam}(\operatorname{supp} \mu)$, and the same properties above hold for $\mathcal{D}_{\mu}:=\bigcup_{j \geq j_{0}} \mathcal{D}_{\mu, j}$.

Given a cube $Q \in \mathcal{D}_{\mu, j}$, we say that its side length is $2^{-j}$, and we denote it by $\ell(Q)$. Notice that $\operatorname{diam}(Q) \leq \ell(Q)$. We also denote

$$
B_{Q}:=B\left(z_{Q}, c_{1} \ell(Q)\right),
$$

where $c_{1}>0$ is some fix constant so that $B_{Q} \cap \operatorname{supp} \mu \subset Q$, for all $Q \in \mathcal{D}_{\mu}$.

For $\lambda>1$, we write

$$
\lambda Q=\{x \in \operatorname{supp} \mu: \operatorname{dist}(x, Q) \leq(\lambda-1) \ell(Q)\} .
$$

2.2. The Riesz transform and harmonic measure. Given a Radon measure $\mu$ in $\mathbb{R}^{n+1}$, its $n$-dimensional Riesz transform is defined by

$$
\mathcal{R} \mu(x)=\int \frac{x-y}{|x-y|^{n+1}} d \mu(y)
$$

whenever the integral makes sense. For $\varepsilon>0$, we also denote

$$
\mathcal{R}_{\varepsilon} \mu(x)=\int_{|x-y|>\varepsilon} \frac{x-y}{|x-y|^{n+1}} d \mu(y), \quad \mathcal{R}_{*} \mu(x)=\sup _{\varepsilon>0}\left|\mathcal{R}_{\varepsilon} \mu(x)\right| .
$$

For $f \in L_{l o c}^{1}(\mu)$, we write $\mathcal{R}_{\mu} f \equiv \mathcal{R}(f \mu), \mathcal{R}_{\mu, \varepsilon} f \equiv \mathcal{R}_{\varepsilon}(f \mu)$, and $\mathcal{R}_{\mu, *} f \equiv \mathcal{R}_{*}(f \mu)$. We say that $\mathcal{R}_{\mu}$ is bounded in $L^{2}(\mu)$ if the operators $\mathcal{R}_{\mu, \varepsilon}$ are bounded in $L^{2}(\mu)$ uniformly on $\varepsilon>0$. We will also use the centered maximal Hardy-Littlewood operator

$$
M_{\mu} f(x)=\sup _{r>0} \frac{1}{\mu(B(x, r))} \int|f| d \mu .
$$

Let $\mathcal{E}$ denote the fundamental solution for the Laplace equation in $\mathbb{R}^{n+1}$, so that $\mathcal{E}(x)=$ $c_{n}|x|^{1-n}$ for $n \geq 2, c_{n}>0$.The Green function $G: \Omega \times \Omega \rightarrow[0, \infty]$ for an open set $\Omega \subset \mathbb{R}^{n+1}$ is a function with the following properties: for each $x \in \Omega$,

$$
G(x, y)=\mathcal{E}(x-y)+h_{x}(y),
$$

where $h_{x}$ is harmonic on $\Omega$, and whenever $v_{x}$ is a nonnegative superharmonic function that is the sum of $\mathcal{E}(x-\cdot)$ and another superharmonic function, then $v_{x} \geq G(x, \cdot)$, from which it follows that $G(x, y)$ is unique ([He, Definition 4.2.3]).

An open subset of $\mathbb{R}^{n+1}$ having Green function is called a Greenian set. By [He, Theorem 4.2.10], all open subsets of $\mathbb{R}^{n+1}$ are Greenian for $n \geq 2$. In the case $n=1$, if $\mathcal{H}^{1}(\partial \Omega)>0$ for example, which is implied by the assumptions of Theorem 1.1 , then $\Omega \subset \mathbb{R}^{2}$ is Greenian. 
We denote by $\omega^{p}$ the harmonic measure in $\Omega$ with pole at $p \in \Omega$. The Green function can be then written as follows (see [AG, Lemma 6.8.1]): for $x, y \in \Omega, x \neq y$

$$
G(x, y)=\mathcal{E}(x-y)-\int_{\partial \Omega} \mathcal{E}(x-z) d \omega^{y}(z) .
$$

Notice that the Riesz transform has kernel

$$
K(x)=c_{n} \nabla \mathcal{E}(x),
$$

for a suitable absolute constant $c_{n}$, so that for $x, p \in \Omega$, by (2.3) and (2.4) we get

$$
\begin{aligned}
\mathcal{R} \omega^{p}(x)=c_{n} \nabla_{x} \int \mathcal{E}(x-y) d \omega^{p}(y) & =c_{n} \nabla_{x}(\mathcal{E}(x-p)-G(x, p)) \\
& =K(x-p)-c_{n} \nabla_{x} G(x, p) .
\end{aligned}
$$

The following is a very standard result, usually known as Bourgain's estimate. See for example $\left[\mathrm{AHM}^{3} \mathrm{TV}\right]$ for more details.

Lemma 2.1. There is $\delta_{0}>0$ depending only on $n \geq 1$ so that the following holds for $\delta \in\left(0, \delta_{0}\right]$. If $\Omega \subsetneq \mathbb{R}^{n+1}$ is a domain, $\xi \in \partial \Omega, r>0$, and $B=B(\xi, r)$, then for all $s>n-1$ and all $x \in \delta B$,

$$
\omega^{x}(B) \gtrsim_{n} \frac{\mathcal{H}_{\infty}^{s}(\partial \Omega \cap \delta B)}{(\delta r)^{s}} .
$$

Remark 2.2. If $\mu$ is some measure supported on $\partial \Omega$ such that $\mu(B(y, r)) \leq C r^{n}$ for all $y$, $r>0$, then from the preceding lemma it follows that

$$
\omega^{x}(B) \gtrsim \frac{\mu\left(\delta_{0} B\right)}{\left(\delta_{0} r\right)^{n}} \quad \text { for all } x \in \delta B .
$$

The next lemma is also standard. See for example [AHM $\left.{ }^{3} \mathrm{TV}\right]$ or [AMT1] for the detailed proof.

Lemma 2.3. Let $\Omega \subsetneq \mathbb{R}^{n+1}, \xi \in \partial \Omega, r>0$ and $B:=B(\xi, r)$. Suppose that there exists $a$ point $x_{B} \in \Omega$ so that the ball $B_{0}:=B\left(x_{B}, r / C\right)$ satisfies $4 B_{0} \subset \Omega \cap B$ for some $C>1$. Then in the case $n \geq 2$ the harmonic measure and Green function of $\Omega$ satisfy

$$
\omega^{x}(B) \gtrsim \omega^{x_{B}}(B) r^{n-1} G\left(x, x_{B}\right) \quad \text { for all } x \in \Omega \backslash B_{0} .
$$

In the case $n=1$, if $\Omega$ is Greenian then

$$
\omega^{x}(B) \gtrsim \omega^{x_{B}}(B)\left|G\left(x, x_{B}\right)-G(x, z)\right| \quad \text { for all } x \in \Omega \backslash B_{0} \text { and } z \in \frac{1}{2} B_{0} .
$$

The implicit constants in (2.8) and (2.9) depend only on $C$ and $n$.

If $\partial \Omega$ is $n$-AD-regular and $0<r(B)<\frac{\delta_{0} \operatorname{diam}(\Omega)}{2}$, then by Lemma 2.3 and (2.7), in the case $n \geq 2$ we have for all $x \in \Omega \backslash 2 B$ and all $y \in B \cap \Omega$,

$$
\omega^{x}\left(2 \delta_{0}^{-1} B\right) \gtrsim r^{n-1} G(x, y)
$$

Analogously, in the case $n=1$ we have for all $x \in \Omega \backslash 2 B$ and $y, z \in B \cap \Omega$,

$$
\omega^{x}\left(2 \delta_{0}^{-1} B\right) \gtrsim r^{n-1}|G(x, y)-G(x, z)|
$$


2.3. Uniform and NTA domains. Following [JK], we say that $\Omega$ satisfies the Harnack chain condition if there is a constant $c$ such that for every $\rho>0, \Lambda \geq 1$, and every pair of points $x_{1}, x_{2} \in \Omega$ with $\operatorname{dist}\left(x_{i}, \partial \Omega\right) \geq \rho$ for $i=1,2$ and $\left|x_{1}-x_{2}\right|<\Lambda \rho$, there is a chain of open balls $B_{1}, \ldots, B_{N} \subset \Omega$, with $N \leq C(\Lambda)$, with $x_{1} \in B_{1}, x_{2} \in B_{N}, B_{k} \cap B_{k+1} \neq \varnothing$ and $\operatorname{dist}\left(B_{k}, \partial \Omega\right) \approx_{c} \operatorname{diam}\left(B_{k}\right)$ for all $k$. The preceding chain of balls is called a "Harnack chain". A connected domain $\Omega \subset \mathbb{R}^{n+1}$ is called a uniform domain if it is a corkscrew domain and satisfies the Harnack chain condition. Finally, $\Omega$ is called an NTA domain, (for "non-tangentially accessible") if if $\Omega$ is a uniform domain and the exterior $\mathbb{R}^{n+1} \backslash \bar{\Omega}$ is a non-empty corkscrew domain.

Let Cap denote logarithmic capacity if $n=1$ and Newtonian capacity if $n \geq 2$. A domain $\Omega \subset \mathbb{R}^{n+1}$ satisfies the capacity density condition (or CDC) if there are $R_{\Omega}>0$ and $c_{\Omega}>0$ such that for any ball $B$ centered on $\partial \Omega$ of radius $r(B) \in\left(0, R_{\Omega}\right)$,

$$
\operatorname{Cap}(B \backslash \Omega) \geq \begin{cases}c_{\Omega} r(B) & \text { if } n=1, \\ c_{\Omega} r(B)^{n-1} & \text { if } n \geq 2 .\end{cases}
$$

If $\partial \Omega$ is $\mathrm{AD}$ regular, then $\Omega$ satisfies the $\mathrm{CDC}$.

\section{THE CORONA DECOMPOSITON FOR HARMONIC MEASURE}

In this section we will show that if either of the assumptions (b) or (c) in Theorem 1.1 holds, then there exists a family $\mathcal{F}$ having the properties described in Theorem 1.3. Later, in Section 5, we will show that the existence of such a family $\mathcal{F}$ implies the uniform rectifiability of $\mu$. The proof of these facts will yield both Theorem 1.1 and 1.3, because Hofmann, Martell and Mayboroda have already shown in [HMM2] that both (b) and (c) in Theorem 1.1 hold if $\mu$ is uniformly rectifiable.

We assume throughout this section that $\Omega \subset \mathbb{R}^{n+1}$ is a corkscrew domain with $n$-ADregular boundary, and that either assumption (b) or (c) of Theorem 1.1 holds. We denote $\mu=\left.\mathcal{H}^{n}\right|_{\partial \Omega}$, and we consider the associated David-Semmes lattice $\mathcal{D}_{\mu}$.

3.1. The corona decomposition. It will be convenient to rephrase the properties of the required family $\mathcal{F}$ in terms of a corona type decomposition. A corona decomposition of $\mu$ is a partition of $\mathcal{D}_{\mu}$ into trees. A family $\mathcal{T} \subset \mathcal{D}_{\mu}$ is a tree if it verifies the following properties:

(1) $\mathcal{T}$ has a maximal element (with respect to inclusion) $Q(\mathcal{T})$ which contains all the other elements of $\mathcal{T}$ as subsets of $\mathbb{R}^{n+1}$. The cube $Q(\mathcal{T})$ is the "root" of $\mathcal{T}$.

(2) If $Q, Q^{\prime}$ belong to $\mathcal{T}$ and $Q \subset Q^{\prime}$, then any $\mu$-cube $P \in \mathcal{D}^{\mu}$ such that $Q \subset P \subset Q^{\prime}$ also belongs to $\mathcal{T}$.

(3) If $Q \in \mathcal{T}$, then either all or none of the children of $Q$ belong to $\mathcal{T}$. If $R=Q(\mathcal{T})$, we also write $\mathcal{T}=\operatorname{Tree}(R)$.

The precise result that we intend to prove in this section is the following.

Proposition 3.1. Let $\Omega \subset \mathbb{R}^{n+1}$ be a corkscrew domain with $n$-AD-regular boundary. Denote by $\mu$ the surface measure on $\partial \Omega$. Suppose that either the assumption $(b)$ or $(c)$ from Theorem 1.1 holds. Then $\mu$ admits a corona decomposition $\mathcal{D}_{\mu}=\bigcup_{R \in \operatorname{Top}} \operatorname{Tree}(R)$ so that 
the family Top is a Carleson family, that is,

$$
\sum_{R \subset S: R \in \text { Top }} \mu(R) \leq C \mu(S) \quad \text { for all } S \in \mathcal{D}_{\mu},
$$

and for each $R \in$ Top there exists a corkscrew point $p_{R} \in \Omega$ with

$$
c^{-1} \ell(R) \leq \operatorname{dist}\left(p_{R}, R\right) \leq \operatorname{dist}\left(p_{R}, \partial \Omega\right) \leq c \ell(R)
$$

so that

$$
\omega^{p_{R}}(3 Q) \approx \frac{\mu(Q)}{\mu(R)} \quad \text { for all } Q \in \operatorname{Tree}(R),
$$

with the implicit constant uniform on $Q$ and $R$.

It is easy to check that the existence of a corona decomposition such as the one in the proposition implies the existence of a family $\mathcal{F} \subset \mathcal{D}_{\mu}$ like the one described in Theorem 1.3. Indeed, if the above corona decomposition exists we just take $\mathcal{F}=$ Top, and we can check that this satisfies the properties stated in Theorem 1.3, since (3.2) also holds with $5 Q$ replaced by $3 Q$ (with a different implicit constant). So Proposition 3.1 proves one of the implications in Theorem 1.3.

3.2. The approximation lemma. For any $Q \in \mathcal{D}_{\mu}$, we consider a corkscrew point $p_{Q} \in$ $B_{Q} \cap \Omega$. Recall that $\omega^{p_{Q}}(Q) \gtrsim 1$, assuming that $p_{Q}$ has been chosen close enough to the center of $Q$, for example. A more quantitative result is the following:

Lemma 3.2. There are constants $0<\alpha<1$ and $c_{2}>0$, depending only on $n$ and the $A D$-regularity constant of $\mu$ such that the following holds. For any $0<\varepsilon<1 / 2$ and any $Q \in \mathcal{D}_{\mu}$, we have

$$
\omega^{x}(Q) \geq \omega^{x}\left(\frac{3}{4} B_{Q}\right) \geq 1-c_{2} \varepsilon^{\alpha} \quad \text { if } x \in \frac{1}{2} B_{Q} \text { and } \operatorname{dist}(x, \partial \Omega) \leq \varepsilon \ell(Q) .
$$

Proof. Since $\omega^{x}\left(\left(\frac{3}{4} B_{Q}\right)^{c}\right)$ is harmonic on $\Omega$, bounded by 1 , and vanishes on $\partial \Omega \cap \frac{3}{4} B_{Q}$, and since $\partial \Omega$ is $n$-AD-regular (and thus $\Omega$ satisfies the CDC), there exists some $\alpha>0$ such that

$$
\omega^{x}\left(\left(\frac{3}{4} B_{Q}\right)^{c}\right) \leq C\left(\frac{\operatorname{dist}(x, \partial \Omega)}{\ell(Q)}\right)^{\alpha}
$$

if $x \in \frac{1}{2} B_{Q}$ (see for example Lemma 4.5 and Corollary 4.6 from [AMT2]). Therefore $\omega^{x}\left(\left(\frac{3}{4} B_{Q}\right)^{c}\right) \lesssim \varepsilon^{\alpha}$ if $\operatorname{dist}(x, \partial \Omega) \leq \varepsilon \ell(Q)$.

From now on, we will assume that $p_{Q} \in \frac{1}{2} B_{Q} \cap \Omega$, with

$$
\operatorname{dist}\left(p_{Q}, \partial \Omega\right) \approx \varepsilon \ell(Q), \quad \varepsilon \ll 1,
$$

so that $\omega^{p_{Q}}(Q) \geq \omega^{x}\left(\frac{3}{4} B_{Q}\right) \geq 1-C \varepsilon^{\alpha}$. The corkscrew condition for $\Omega$ ensures the existence of such point $p_{Q}$. We denote by $y_{Q}$ a point in $\partial \Omega$ such that

$$
\operatorname{dist}\left(p_{Q}, \partial \Omega\right)=\left|y_{Q}-p_{Q}\right|,
$$

and we assume that $p_{Q}$ has been chosen so that

$$
B\left(y_{Q},\left|y_{Q}-p_{Q}\right|\right) \subset \frac{3}{4} B_{Q} .
$$

We also denote $V_{Q}=B\left(p_{Q}, \frac{1}{10} \operatorname{dist}\left(p_{Q}, \partial \Omega\right)\right)$, so that $V_{Q} \subset \Omega$. Notice that

$$
r\left(V_{Q}\right) \approx \varepsilon \ell(Q) \text {. }
$$


The next lemma is the main technical result of this subsection.

Lemma 3.3. Suppose that the constant $\varepsilon$ in (3.3) is small enough. Let $Q \in \mathcal{D}_{\mu}$ and let $E_{Q} \subset Q$ be such that

$$
\omega^{p_{Q}}\left(E_{Q}\right) \geq(1-\varepsilon) \omega^{p_{Q}}(Q) .
$$

Then there exists a non-negative harmonic function $u_{Q}$ on $\Omega$ and a Borel function $f_{Q}$ with

$$
\begin{gathered}
u_{Q}(x)=\int_{E_{Q}} f_{Q} d \omega^{x}, \\
f_{Q} \leq c \chi_{E_{Q}} \text { if } n \geq 2, \quad \text { and } \quad f_{Q} \leq c|\log \varepsilon| \chi_{E_{Q}} \text { if } n=1,
\end{gathered}
$$

and a unit vector $e_{Q} \in \mathbb{R}^{n+1}$ such that

$$
\nabla u_{Q}(x) \cdot e_{Q} \geq c \frac{1}{r\left(V_{Q}\right)} \quad \text { for all } x \in V_{Q} .
$$

In particular,

$$
\int_{V_{Q}}\left|\nabla u_{Q}(x)\right|^{2} \operatorname{dist}(x, \partial \Omega) d x \gtrsim r\left(V_{Q}\right)^{n} \approx_{\varepsilon} \ell(Q)^{n} .
$$

Proof in the case $n \geq 2$. Let $y_{Q} \in \partial \Omega$ be the point defined in (3.4). By rotating the domain if necessary we may assume that $p_{Q}-y_{Q}$ is parallel to the $x$ axis and that $p_{Q, 1}>y_{Q, 1}$. Then, for all $x \in V_{Q}$ and all $y \in B\left(y_{Q}, r\left(V_{Q}\right)\right)$,

$$
0<\frac{x_{1}-y_{1}}{|x-y|^{n+1}} \approx \frac{1}{r\left(V_{Q}\right)^{n}}
$$

Therefore, if we take

$$
g_{Q}(x):=\int_{B\left(y_{Q}, r\left(V_{Q}\right)\right)} \frac{1}{r\left(V_{Q}\right)|x-y|^{n-1}} d \mu(y) \quad \text { if } n \geq 2,
$$

then we have

$$
\begin{aligned}
\left|\nabla g_{Q}(x)\right| & \geq-\partial_{1} g_{Q}(x)=c \int_{B\left(y_{Q}, r\left(V_{Q}\right)\right)} \frac{x_{1}-y_{1}}{r\left(V_{Q}\right)|x-y|^{n+1}} d \mu(y) \\
& \approx \frac{\mu\left(B\left(y_{Q}, r\left(V_{Q}\right)\right)\right.}{r\left(V_{Q}\right)^{n+1}} \approx \frac{1}{r\left(V_{Q}\right)} \quad \text { for all } x \in V_{Q} .
\end{aligned}
$$

By the AD-regularity of $\mu$, it is also immediate that $\left\|g_{Q}\right\|_{\infty} \lesssim 1$. Then we define $f_{Q}:=$ $\chi_{E_{Q}} g_{Q}$ and

$$
u_{Q}(x):=\int f_{Q} d \omega^{x}=\int_{E_{Q}} g_{Q} d \omega^{x} .
$$

To prove the estimate (3.6) with $e_{Q}=-e_{1}$, first note that $g_{Q}$ is harmonic in $\Omega$ and continuous in $\mathbb{R}^{n+1}$, because of the local $\mu$ uniform integrability of $1 /|x-y|^{n-1}$. Thus, for all $x \in \Omega$,

$$
g_{Q}(x)=\int g_{Q} d \omega^{x},
$$

and then,

$$
\left|g_{Q}(x)-u_{Q}(x)\right|=\left|\int_{\partial \Omega \backslash E_{Q}} g_{Q} d \omega^{x}\right| \leq\left\|g_{Q}\right\|_{\infty} \omega^{x}\left(\partial \Omega \backslash E_{Q}\right) \lesssim \omega^{x}\left(\partial \Omega \backslash E_{Q}\right) .
$$


By (3.3) and the assumption in the lemma,

$$
\omega^{p_{Q}}\left(\partial \Omega \backslash E_{Q}\right)=\omega^{p_{Q}}(\partial \Omega \backslash Q)+\omega^{p_{Q}}\left(Q \backslash E_{Q}\right) \leq C \varepsilon^{\alpha}+\varepsilon \lesssim \varepsilon^{\alpha},
$$

and then by Harnack's inequality it follows that

$$
\omega^{x}\left(\partial \Omega \backslash E_{Q}\right) \lesssim \varepsilon^{\alpha} \quad \text { for all } x \in 2 V_{Q} .
$$

Therefore,

$$
\left|g_{Q}(x)-u_{Q}(x)\right| \lesssim \varepsilon^{\alpha} \quad \text { for all } x \in 2 V_{Q} .
$$

Since $g_{Q}-u_{Q}$ is harmonic, we have

$$
\left|\nabla\left(g_{Q}-u_{Q}\right)(x)\right| \lesssim \frac{1}{r\left(V_{Q}\right)} f_{2 V_{Q}}\left|g_{Q}-u_{Q}\right| d y \lesssim \varepsilon^{\alpha} \frac{1}{r\left(V_{Q}\right)} \quad \text { for all } x \in V_{Q},
$$

and so, assuming $\varepsilon$ small enough,

$$
-\partial_{1} u_{Q}(x) \geq-\partial_{1} g_{Q}(x)-\left|\nabla\left(g_{Q}-u_{Q}\right)(x)\right| \gtrsim \frac{1}{r\left(V_{Q}\right)} \quad \text { for all } x \in V_{Q},
$$

which concludes the proof of (3.6).

The final estimate (3.7) is an immediate consequence of (3.6).

Proof of Lemma 3.3 in the case $n=1$. As above, we assume that $p_{Q}-y_{Q}$ is parallel to the $x$ axis and that $p_{Q, 1}>y_{Q, 1}$, so that

$$
0<\frac{x_{1}-y_{1}}{|x-y|^{2}} \approx \frac{1}{r\left(V_{Q}\right)} \quad \text { for all } x \in V_{Q} \text { and all } y \in B\left(y_{Q}, r\left(V_{Q}\right)\right) \text {. }
$$

We now define a function $g_{Q}$ which will play the role of the analogous one in (3.8). To this end, note that because of the AD-regularity of $\mu$ there exists some point

$$
y_{2} \in \operatorname{supp} \mu \cap A\left(y_{Q}, 3 \ell(Q), 4 c_{0}^{2} \ell(Q)\right),
$$

where $A\left(x, r_{1}, r_{2}\right)$ stands for the open annulus centered at $x$ with inner radius $r_{1}$ and outer radius $r_{2}$, and $c_{0}$ is the AD-regularity constant of $\mu$. Consider a ball $B_{2}$ centered at $y_{2}$ with radius $r\left(V_{Q}\right)$. To shorten notation we write $B_{1}=B\left(y_{Q}, r(V(Q)), r=r\left(V_{Q}\right)\right.$, and $y_{1}=y_{Q}$. Then we define

$$
g_{Q}(x)=\int_{B_{1}} \frac{1}{r} \log \frac{1}{|x-y|} d \mu(y)-\frac{\mu\left(B_{1}\right)}{\mu\left(B_{2}\right)} \int_{B_{2}} \frac{1}{r} \log \frac{1}{|x-y|} d \mu(y) .
$$

We claim that $g_{Q}$ satisfies the following properties:

(a) $g_{Q} \in C_{0}(\bar{\Omega})$,

(b) $g_{Q} \geq 0$ on $Q$ and $\left\|g_{Q}\right\|_{\infty} \lesssim|\log \varepsilon|$,

(c) $0 \leq-\partial_{1} g_{Q}(x) \approx \frac{1}{r\left(V_{Q}\right)}$ for all $x \in V_{Q}$.

Using the properties above and arguing as in the case $n \geq 2$, we can complete the proof of the lemma in this case. Indeed, from (3.9), taking account that $\omega^{p_{Q}}\left(\partial \Omega \backslash E_{Q}\right) \lesssim \varepsilon^{\alpha}$ (because (3.10) is still valid) and that $\left\|g_{Q}\right\|_{\infty} \lesssim|\log \varepsilon|$, we deduce that

$$
\left|g_{Q}(x)-u_{Q}(x)\right| \leq \varepsilon^{\alpha}|\log \varepsilon| \text { for all } x \in V_{Q} .
$$


Then, as in (3.11) we derive that

$$
\left|\nabla\left(g_{Q}-u_{Q}\right)(x)\right| \lesssim \varepsilon^{\alpha}|\log \varepsilon| \frac{1}{r\left(V_{Q}\right)} \quad \text { for all } x \in V_{Q},
$$

which together with the property (c) above yields (3.6), for $\varepsilon$ small enough.

Again, the final estimate (3.7) is an immediate consequence of (3.6).

We now verify the claims (a), (b) and (c). The continuity of $g_{Q}$ follows easily from the local $\mu$ uniform integrability of the kernel $\log \frac{1}{|x-y|}$. To see that it vanishes at infinity, note that $g_{Q}$ can be written as follows:

$$
g_{Q}(x)=\int_{B_{1}} \frac{1}{r} \log \frac{\left|x-y_{1}\right|}{|x-y|} d \mu(y)-\frac{\mu\left(B_{1}\right)}{\mu\left(B_{2}\right)} \int_{B_{2}} \frac{1}{r} \log \frac{\left|x-y_{1}\right|}{|x-y|} d \mu(y),
$$

and then

$$
\log \frac{\left|x-y_{1}\right|}{|x-y|} \rightarrow 0 \quad \text { as } x \rightarrow \infty .
$$

To show that $g_{Q} \geq 0$ on $Q$, write

$$
g_{Q}(x)=\int_{B_{1}} \frac{1}{r} \log \frac{\ell(Q)}{|x-y|} d \mu(y)-\frac{\mu\left(B_{1}\right)}{\mu\left(B_{2}\right)} \int_{B_{2}} \frac{1}{r} \log \frac{\ell(Q)}{|x-y|} d \mu(y)=: g_{1}(x)-g_{2}(x) \text {. }
$$

Observe that

$$
\frac{\ell(Q)}{|x-y|}>1 \quad \text { for all } y \in B_{1}
$$

while

$$
\frac{\ell(Q)}{|x-y|}<1 \quad \text { for all } y \in B_{2} \text {. }
$$

So $g_{1}(x)>0$ and $g_{2}(x)<0$ for $x \in Q$, and thus $g_{Q}(x)>0$ on $Q$.

To estimate $\left\|g_{Q}\right\|_{\infty}$, suppose first that $x \notin B\left(y_{1}, 10 c_{0}^{2} \ell(Q)\right)$. For these points $x$ we have

$$
|x-y| \approx\left|x-y_{1}\right| \approx\left|x-y_{2}\right| \quad \text { for all } y \in B_{1} \cup B_{2} .
$$

So

$$
-C \leq \log \frac{\left|x-y_{1}\right|}{|x-y|} \leq C \quad \text { for } x \notin B\left(y_{1}, 10 c_{0}^{2} \ell(Q)\right) \text { and } y \in B_{1} \cup B_{2} . .
$$

Then, from the identity (3.14), taking into account that $\mu\left(B_{1}\right) \approx \mu\left(B_{2}\right)$ we deduce

$$
\left|g_{Q}(x)\right| \lesssim \frac{\mu\left(B_{1}\right)}{r}+\frac{\mu\left(B_{2}\right)}{r} \lesssim 1 \text { for } x \notin B\left(y_{1}, 10 c_{0}^{2} \ell(Q)\right) .
$$

In the case $x \in B\left(y_{1}, 10 c_{0}^{2} \ell(Q)\right)$ we write

$$
g_{Q}(x)=\int_{B_{1}} \frac{1}{r} \log \frac{r}{|x-y|} d \mu(y)-\frac{\mu\left(B_{1}\right)}{\mu\left(B_{2}\right)} \int_{B_{2}} \frac{1}{r} \log \frac{r}{|x-y|} d \mu(y)=: \widetilde{g}_{1}(x)-\widetilde{g}_{2}(x) \text {. }
$$

Let us estimate $\widetilde{g}_{1}(x)$. To this end, note first that if $x \in B\left(y_{1}, 10 c_{0}^{2} \ell(Q)\right) \backslash 2 B_{1}$, then

$$
1 \leq \frac{|x-y|}{r} \lesssim \frac{\ell(Q)}{r} \approx \frac{1}{\varepsilon} .
$$

Hence $\left|\log \frac{r}{|x-y|}\right| \lesssim|\log \varepsilon|$ and thus $\left|\widetilde{g}_{1}(x)\right| \lesssim|\log \varepsilon|$. On the other hand, if $x \in 2 B_{1}$, then

$$
\left|\widetilde{g}_{1}(x)\right| \leq \int_{B(x, 4 r)} \frac{1}{r}\left|\log \frac{r}{|x-y|}\right| d \mu(y) .
$$


By the linear growth of $\mu$ it easy to check that the last integral is bounded above by some constant depending only on the growth constant for $\mu$. So in any case we have $\left|\widetilde{g}_{1}(x)\right| \lesssim$ $|\log \varepsilon|$ for $x \in B\left(y_{1}, 10 c_{0}^{2} \ell(Q)\right)$. The same estimate holds for $\widetilde{g}_{2}(x)$, and then it follows that

$$
\left|g_{Q}(x)\right| \lesssim|\log \varepsilon| \quad \text { for all } x \in B\left(y_{1}, 10 c_{0}^{2} \ell(Q)\right),
$$

which concludes the proof of $\left\|g_{Q}\right\|_{\infty} \lesssim|\log \varepsilon|$.

Finally we verify (c). We have

$$
-\partial_{1} g_{Q}(x)=\int_{B_{1}} \frac{1}{2 r} \frac{x_{1}-y_{1}}{|x-y|^{2}} d \mu(y)-\frac{\mu\left(B_{1}\right)}{\mu\left(B_{2}\right)} \int_{B_{2}} \frac{1}{2 r} \frac{x_{1}-y_{1}}{|x-y|^{2}} d \mu(y)=: h_{1}(x)-h_{2}(x) \text {. }
$$

From (3.12) we get

$$
0<h_{1}(x) \approx \frac{1}{r\left(V_{Q}\right)} \quad \text { for all } x \in V_{Q},
$$

while taking into account that $\operatorname{dist}\left(V_{Q}, B_{2}\right) \approx \ell(Q)$, we have

$$
\left|h_{2}(x)\right| \lesssim \frac{1}{\ell(Q)} \quad \text { for all } x \in V_{Q},
$$

Thus

$$
-\partial_{1} g_{Q}(x) \gtrsim \frac{1}{r\left(V_{Q}\right)}-\frac{c}{\ell(Q)} \approx \frac{1}{r\left(V_{Q}\right)},
$$

and so the proof of the claim is concluded.

3.3. The stopping cubes. Before defining the family Top, we need to define, for any given $R \in \mathcal{D}_{\mu}$, two associated families $\operatorname{HD}(R)$ and $\operatorname{LD}(R)$ of high density and low density cubes, respectively.

Let $0<\delta \ll 1$ and $A \gg 1$ be some fixed constants. For a fixed a cube $R \in \mathcal{D}_{\mu}$, let $Q \in \mathcal{D}_{\mu}, Q \subset R$. We say that $Q \in \operatorname{HD}(R)$ (high density) if $Q$ is a maximal cube satisfying

$$
\frac{\omega^{p_{R}}(2 Q)}{\mu(2 Q)} \geq A \frac{\omega^{p_{R}}(2 R)}{\mu(2 R)} .
$$

We say that $Q \in \operatorname{LD}(R)$ (low density) if $Q$ is a maximal cube satisfying

$$
\frac{\omega^{p_{R}}(Q)}{\mu(Q)} \leq \delta \frac{\omega^{p_{R}}(R)}{\mu(R)}
$$

(notice that $\omega^{p_{R}}(R) \approx \omega^{p_{R}}(2 R) \approx 1$ by (2.6)). Observe that the definition of the family $\mathrm{HD}(R)$ involves the density of $2 Q$, while the one of $\operatorname{LD}(R)$ involves the density of $Q$.

We denote

$$
B_{H}(R)=\bigcup_{Q \in \mathrm{HD}(R)} Q \quad \text { and } \quad B_{L}(R)=\bigcup_{Q \in \mathrm{LD}(R)} Q
$$

Lemma 3.4. We have

$$
\mu\left(B_{H}(R)\right) \lesssim \frac{1}{A} \mu(R)
$$


Proof. By Vitali's covering theorem, there exists a subfamily $I \subset \mathrm{HD}(R)$ so that the cubes $2 Q, Q \in I$, are pairwise disjoint and

$$
\bigcup_{Q \in \mathrm{HD}(R)} 2 Q \subset \bigcup_{Q \in I} 6 Q
$$

Then, using that $\mu$ is doubling,

$$
\mu\left(B_{H}(R)\right) \lesssim \sum_{Q \in I} \mu(2 Q) \leq \frac{1}{A} \sum_{Q \in I} \frac{\omega^{p_{R}}(2 Q)}{\omega^{p_{R}}(2 R)} \mu(2 R) \lesssim \frac{1}{A} \mu(R) .
$$

Concerning the low density cubes, we have:

Lemma 3.5. We have

$$
\omega^{p_{R}}\left(B_{L}(R)\right) \leq \delta \omega^{p_{R}}(R) .
$$

Proof. Since the cubes from $\operatorname{LD}(R)$ are pairwise disjoint, we have

$$
\omega^{p_{R}}\left(B_{L}(R)\right)=\sum_{Q \in \mathrm{LD}(R)} \omega^{p_{R}}(Q) \leq \delta \sum_{Q \in \operatorname{LD}(R)} \frac{\mu(Q)}{\mu(R)} \omega^{p_{R}}(R) \leq \delta \omega^{p_{R}}(R) .
$$

3.4. The family $\operatorname{Top}\left(R_{0}\right)$ and the trees of the corona decomposition. In this subsection, we define, for each $R_{0} \in \mathcal{D}_{\mu}$, a localized version of the family Top, which we will denote by $\operatorname{Top}\left(R_{0}\right)$. To this end, given a cube $R \in \mathcal{D}_{\mu}$ we let

$$
\operatorname{Stop}(R):=\{S \in \mathrm{HD}(R) \cup \operatorname{LD}(R): \nexists \widetilde{S} \in \operatorname{HD}(R) \cup \operatorname{LD}(R) \text { such that } S \subsetneq \widetilde{S}\} .
$$

Notice that by maximality with respect to the inclusion in $\operatorname{HD}(R) \cup \operatorname{LD}(R)$, Stop $(R)$ is a family of pairwise disjoint cubes. We define

$$
\operatorname{Tree}(R):=\left\{Q \in \mathcal{D}_{\mu}(R): \nexists S \in \operatorname{Stop}(R) \text { such that } Q \subsetneq S\right\} .
$$

In particular, note that $\operatorname{Stop}(R) \subset \operatorname{Tree}(R)$. We also define

$$
\widetilde{\operatorname{Stop}}(R):=\left\{Q \in \mathcal{D}_{\mu}(R): \exists S \in \operatorname{Stop}(R) \text { such that } Q \in \operatorname{ch}(S)\right\},
$$

where $\operatorname{ch}(S)$ stands for the children of $S$. Notice that this family is also pairwise disjoint.

We fix a cube $R_{0} \in \mathcal{D}_{\mu}$ and we define the family of the top cubes with respect to $R_{0}$ as follows: first we define the families $\operatorname{Top}_{k}\left(R_{0}\right)$ for $k \geq 0$ inductively. We set

$$
\operatorname{Top}_{0}\left(R_{0}\right)=\left\{R_{0}\right\} \text {. }
$$

Assuming that $\operatorname{Top}_{k}\left(R_{0}\right)$ has been defined, we set

$$
\operatorname{Top}_{k+1}\left(R_{0}\right)=\underset{R \in \operatorname{Top}_{k}\left(R_{0}\right)}{ } \widetilde{\operatorname{Stop}}(R),
$$

and then we define

$$
\operatorname{Top}\left(R_{0}\right)=\bigcup_{k \geq 0} \operatorname{Top}_{k}\left(R_{0}\right)
$$

Notice that

$$
\mathcal{D}_{\mu}\left(R_{0}\right)=\bigcup_{R \in \operatorname{Top}\left(R_{0}\right)} \operatorname{Tree}(R),
$$


and this union is disjoint.

We denote by $\operatorname{Top}_{H}\left(R_{0}\right)$ the subfamily of the cubes from $\operatorname{Top}\left(R_{0}\right)$ whose parents belong to $\operatorname{HD}(R)$ for some $R \in \operatorname{Top}\left(R_{0}\right)$, and by $\operatorname{Top}_{L}\left(R_{0}\right)$ the subfamily of the cubes from $\operatorname{Top}\left(R_{0}\right)$ whose parents belong to $\operatorname{LD}(R)$ for some $R \in \operatorname{Top}\left(R_{0}\right)$. So we have

$$
\operatorname{Top}\left(R_{0}\right)=\left\{R_{0}\right\} \cup \operatorname{Top}_{H}\left(R_{0}\right) \cup \operatorname{Top}_{L}\left(R_{0}\right) .
$$

Observe also that if $Q \in \operatorname{Top}_{H}\left(R_{0}\right)$ (resp. Top $\left.{ }_{L}\left(R_{0}\right)\right)$, then any sibling of $R$ also belongs to $\operatorname{Top}_{H}\left(R_{0}\right)$ (resp. $\left.\operatorname{Top}_{L}\left(R_{0}\right)\right)$.

Lemma 3.6. For any $R \in \operatorname{Top}\left(R_{0}\right)$, the following hods:

$$
\omega^{p_{R}}(3 Q) \approx_{\delta, A} \frac{\mu(Q)}{\mu(R)} \quad \text { for all } Q \in \operatorname{Tree}(R) .
$$

Proof. Let $\widehat{Q} \subset \mathcal{D}_{\mu}$ be the parent of $Q$. It is immediate to check that $\widehat{Q} \subset 3 Q \subset 2 \widehat{Q}$. If $\widehat{Q} \subset R$, by construction

$$
\omega^{p_{R}}(3 Q) \leq \omega^{p_{R}}(2 \widehat{Q}) \leq A \omega^{p_{R}}(2 R) \frac{\mu(2 Q)}{\mu(2 R)} \approx A \frac{\mu(Q)}{\mu(R)},
$$

and also

$$
\omega^{p_{R}}(3 Q) \geq \omega^{p_{R}}(\widehat{Q}) \geq \delta \omega^{p_{R}}(R) \frac{\mu(Q)}{\mu(R)} \approx \delta \frac{\mu(Q)}{\mu(R)} .
$$

If $\widehat{Q} \not \subset R$, then $\widehat{Q}$ is the parent of $R$ and thus $3 Q \supset R$, which implies that $\omega^{p_{R}}(3 Q) \approx 1$ and $\mu(Q) \approx \mu(R)$. Hence the estimate in the lemma is trivially true.

3.5. The iterative construction and the key lemma. Our next goal is to prove that the family $\operatorname{Top}\left(R_{0}\right)$ satisfies a packing condition analogous to the one stated in (5.1) for the family Top. The proof would be easy if the inequality $\left.\mu\left(B_{L}(R)\right)\right) \ll \mu(R)$ followed from Lemma 3.5, but we are unable to verify that. In this subsection we instead prove a variant of the above inequality for $B_{L}^{m}(R)$ for some $m \geq 1$. The set $B_{L}^{m}(R)$ is defined as follows.

For $R \in \mathcal{D}_{\mu}$, we denote $\operatorname{LD}^{0}(R)=\{R\}, \operatorname{LD}^{1}(R)=\operatorname{LD}(R)$, and for $k \geq 1$ we consider the families of cubes

and the subset of $R$ given by

$$
\operatorname{LD}^{k+1}(R)=\bigcup_{Q \in \operatorname{LD}^{k}(R)} \operatorname{LD}(Q),
$$

$$
B_{L}^{k}(R)=\bigcup_{Q \in \mathrm{LD}^{k}(R)} Q .
$$

Notice that the stopping conditions in the definition of the family of low density cubes $\operatorname{LD}^{k}(R)$ involve the harmonic measure $\omega^{p_{Q}}$ for a suitable $Q \in \operatorname{LD}^{k-1}(R)$, instead of $\omega^{p_{R}}$.

The next lemma is one of the key steps for the proof of Theorem 1.1.

Lemma 3.7 (Key Lemma). Suppose that either the assumption (b) or (c) in Theorem 1.1 holds. Suppose also that $\varepsilon$ in (3.3) is chosen small enough and that $\delta \leq \varepsilon$. Then for any $m \geq 1$ we have

$$
\sum_{k=1}^{m} \sum_{Q \in \mathrm{LD}^{k}(R)} \mu(Q) \lesssim_{\varepsilon} \mu(R)
$$


and

$$
\mu\left(B_{L}^{m}(R)\right) \lesssim \varepsilon \frac{1}{m} \mu(R) .
$$

Proof. For $Q \subset \mathcal{D}_{\mu}, Q \subset R$, we denote

$$
E_{Q}=Q \backslash B_{L}(Q)
$$

By Lemma 3.2 and Lemma 3.5 applied to $Q$,

$$
\begin{gathered}
\omega^{p_{Q}}\left(E_{Q}\right)=\omega^{p_{Q}}(Q)-\omega^{p_{Q}}\left(B_{L}(Q)\right) \geq \\
\geq(1-\delta) \omega^{p_{Q}}(Q) \geq(1-\delta)\left(1-c \varepsilon^{\alpha}\right) \geq 1-c^{\prime} \varepsilon^{\alpha} .
\end{gathered}
$$

Hence, by Lemma 3.3, if $\varepsilon$ is small enough and $\delta \leq \varepsilon$, there exists a function $u_{Q}$ on $\Omega$ and a non-negative Borel function $f_{Q}$ with $^{1} f_{Q} \leq c|\log \varepsilon| \chi_{E_{Q}}$ such that

$$
u_{Q}(x)=\int_{E_{Q}} f_{Q} d \omega^{x}
$$

satisfying, for some unit vector $e_{Q} \in \mathbb{R}^{n+1}$,

$$
\nabla u_{Q}(x) \cdot e_{Q} \geq c \frac{1}{r\left(V_{Q}\right)} \quad \text { for all } x \in V_{Q},
$$

and so that

$$
\int_{V_{Q}}\left|\nabla u_{Q}(x)\right|^{2} \operatorname{dist}(x, \partial \Omega) d x \geq \operatorname{cr}\left(V_{Q}\right)^{n} .
$$

Notice that the set $E_{Q}$ is disjoint form the low density cubes from $\operatorname{LD}(Q)$, so that by construction the sets $E_{Q}, Q \in \mathrm{LD}^{k}(R), k \geq 1$, are pairwise disjoint. This implies that the function

$$
u:=\sum_{k=1}^{m} \sum_{Q \in \mathrm{LD}^{k}(R)} u_{Q}
$$

is uniformly bounded by $c|\log \varepsilon|$ on $\Omega$. Indeed, by the definitions of the functions $u$ and $u_{Q}$,

$$
\begin{aligned}
u(x) & =\int \sum_{k=1}^{m} \sum_{Q \in \mathrm{LD}^{k}(R)} f_{Q} \chi_{E_{Q}} d \omega^{x} \\
& \leq c|\log \varepsilon| \sum_{k=1}^{m} \sum_{Q \in \mathrm{LD}^{k}(R)} \omega^{x}\left(E_{Q}\right) \leq c|\log \varepsilon| .
\end{aligned}
$$

Remark that the latter estimate also holds with $u$ replaced by $u-u_{Q}$.

We claim that for all $x \in V_{Q}$, for $Q \in \operatorname{LD}^{k}(R), k \geq 1$,

$$
|\nabla u(x)| \geq \nabla u(x) \cdot e_{Q} \gtrsim \frac{1}{r\left(V_{Q}\right)} .
$$

To show this, we set

$$
\nabla u(x) \cdot e_{Q} \geq \nabla u_{Q}(x) \cdot e_{Q}-\left|\nabla\left(u-u_{Q}\right)(x)\right| \geq \frac{c}{r\left(V_{Q}\right)}-\left|\nabla\left(u-u_{Q}\right)(x)\right| .
$$

\footnotetext{
${ }^{1}$ In the case $n \geq 2$ this can be improved to $f_{Q} \leq c \chi_{E_{Q}}$, but we will not use this.
} 
Since $u-u_{Q}$ is harmonic and positive in $3 V_{Q}$, we have

$$
\left|\nabla\left(u-u_{Q}\right)(x)\right| \lesssim \frac{1}{r\left(V_{Q}\right)}\left\|u-u_{Q}\right\|_{L^{\infty}\left(2 V_{Q}\right)} \approx \frac{1}{r\left(V_{Q}\right)}\left(u-u_{Q}\right)\left(p_{Q}\right) .
$$

Now, since $u-u_{Q}$ is harmonic in $\Omega$ and vanishes in $E_{Q}$ we obtain

$$
\left(u-u_{Q}\right)\left(p_{Q}\right)=\int\left(u-u_{Q}\right) d \omega^{p_{Q}} \leq\left\|u-u_{Q}\right\|_{L^{\infty}(\partial \Omega)} \omega^{p_{Q}}\left(\partial \Omega \backslash E_{Q}\right) \leq C|\log \varepsilon| \varepsilon^{\alpha},
$$

where in the last inequality we used (3.17) along with (3.18) for $u-u_{Q}$. Hence

$$
\nabla u(x) \cdot e_{Q} \geq \frac{c}{r\left(V_{Q}\right)}-\frac{C|\log \varepsilon| \varepsilon^{\alpha}}{r\left(V_{Q}\right)}
$$

and our claim follows if $\varepsilon$ is taken small enough.

- Suppose first that the assumption (c) in Theorem 1.1 holds. From the claim (3.19) and the fact that the sets $V_{Q}, Q \in \operatorname{LD}^{k}(R), k \geq 1$, are pairwise disjoint (or at least, have bounded overlap), we get

$$
\begin{aligned}
\int_{B(R)}|\nabla u|^{2} \operatorname{dist}(x, \partial \Omega) d x & \gtrsim \sum_{k=1}^{m} \sum_{Q \in \mathrm{LD}^{k}(R)} \int_{V_{Q}}|\nabla u(x)|^{2} \operatorname{dist}(x, \partial \Omega) d x \\
& \gtrsim \sum_{k=1}^{m} \sum_{Q \in \mathrm{LD}^{k}(R)} r\left(V_{Q}\right)^{n} \approx_{\varepsilon} \sum_{k=1}^{m} \sum_{Q \in \mathrm{LD}^{k}(R)} \mu(Q),
\end{aligned}
$$

where $B(R)$ is some big ball concentric with $R$, with radius comparable to $\ell(R)$, which contains the sets $V_{Q}, Q \in \operatorname{LD}^{k}(R), k=1, \ldots, m$. Then, from (1.4) we derive

$$
\sum_{k=1}^{m} \sum_{Q \in \mathrm{LD}^{k}(R)} \mu(Q) \lesssim_{\varepsilon} \mu(R),
$$

which yields the first assertion of the lemma in this case.

- Suppose now that the hypothesis (b) in Theorem 1.1 holds, i.e., that for all $\varepsilon_{0}>0$ every bounded harmonic function on $\Omega$ is $\varepsilon_{0}$-approximable. So, for some $\varepsilon_{0}>0$ small enough to be chosen below, let $\varphi \in W_{\text {loc }}^{1,1}(\Omega)$ such that $\|u-\varphi\|_{L^{\infty}(\Omega)}<\varepsilon_{0}$ and

$$
\int_{B(R)}|\nabla \varphi(y)| d y \leq C \mu(R),
$$

where $B(R)$ is as above. We claim that

$$
\int_{V_{Q}}|\nabla \varphi(y)| d y \gtrsim \varepsilon \ell(Q)^{n} \quad \text { for all } Q \in \operatorname{LD}^{k}(R), k=1, \ldots, m .
$$

Indeed, for each such $Q$ consider two balls $V_{Q}^{1}, V_{Q}^{2} \subset V_{Q}$ such that $r\left(V_{Q}^{1}\right)=r\left(V_{Q}^{1}\right)=$ $\frac{1}{100} r\left(V_{Q}\right)$, and so that $V_{Q}^{2}=\frac{r\left(V_{Q}\right)}{10} e_{Q}+V_{Q}^{1}$ (i.e., $V_{Q}^{2}$ is the translation of $V_{Q}^{1}$ by the vector $\left.\frac{r\left(V_{Q}\right)}{10} e_{Q}\right)$. Then, by a change of variable, the mean value theorem, and (3.19) it follows that

$$
\begin{aligned}
f_{V_{Q}^{2}} u(y) d y-f_{V_{Q}^{1}} u(y) d y & =f_{V_{Q}^{1}}\left(u\left(y+\frac{r\left(V_{Q}\right)}{10} e_{Q}\right)-u(y)\right) d y \\
& \geq \operatorname{cr}\left(V_{Q}\right) \min _{y \in V_{Q}}\left[\nabla u(y) \cdot e_{Q}\right] \gtrsim 1 .
\end{aligned}
$$


Hence, if $\varepsilon_{0}$ is small enough, then we also have

$$
f_{V_{Q}^{2}} \varphi(y) d y-f_{V_{Q}^{1}} \varphi(y) d y \gtrsim 1 .
$$

Then (3.22) is an immediate consequence of the Poincaré inequality applied to the ball $V_{Q}$.

Arguing as in (3.20), from (3.22) and (3.21) we deduce

$$
\sum_{k=1}^{m} \sum_{Q \in \mathrm{LD}^{k}(R)} \mu(Q) \lesssim \varepsilon \int_{B(R)}|\nabla \varphi(y)| d y \lesssim \mu(R),
$$

which completes the proof of the first assertion of the lemma.

The second estimate in the lemma follows from the fact that if $Q \in B_{L}^{m}(R)$, then $x$ belongs to $m$ different cubes $Q \in \operatorname{LD}^{k}(R), k=1, \ldots, m$, so that

$$
\sum_{k=1}^{m} \sum_{Q \in \mathrm{LD}^{k}(R)} \chi_{Q}(x)=m
$$

and by Chebyshev,

$$
\mu\left(B_{L}^{m}(R)\right) \leq \frac{1}{m} \sum_{k=1}^{m} \sum_{Q \in \mathrm{LD}^{k}(R)} \mu(Q) \lesssim \varepsilon \frac{1}{m} \mu(R) .
$$

Remark 3.8. The preceding lemma also holds if we assume that either the assumption (b) or (c) in Theorem 1.1 is only satisfied by functions $u \in C(\bar{\Omega})$ which are bounded and harmonic in $\Omega$. The proof is almost the same. Indeed, consider a finite subfamily $\mathcal{L} \subset \bigcup_{k=0}^{m} \operatorname{LD}^{k}(R)$. For each $Q \in \mathcal{L}$, set

$$
\widetilde{E}_{Q}=\frac{3}{4} B_{Q} \backslash \bigcup_{P \in \mathcal{L}: P \subsetneq Q} P .
$$

Arguing as in (3.17), we deduce that $\omega^{p_{Q}}\left(\widetilde{E}_{Q}\right) \geq 1-c \varepsilon^{\alpha}$. Consider a continuous function $\varphi_{Q}$ such that $0 \leq \varphi_{Q} \leq 1$ with

$$
\varphi_{Q}(x)= \begin{cases}1 & \text { if } x \in \widetilde{E}_{Q}, \\ 0 & \text { if } x \in\left(\frac{4}{5} B_{Q}\right)^{c} \cup \bigcup_{P \in \mathcal{L}: P \subsetneq Q} \frac{4}{5} B_{P} .\end{cases}
$$

Set

$$
\widetilde{u}_{Q}=\int \varphi_{Q} g_{Q} d \omega^{x}
$$

where $g_{Q}$ is defined in (3.8) and (3.13). Since both $\varphi_{Q}$ and $g_{Q}$ are continuous in $\bar{\Omega}$, it follows easily that $\widetilde{u}_{Q} \in C_{0}(\bar{\Omega})$. Consider the function $\widetilde{u}:=\sum_{Q \in \mathcal{L}} \widetilde{u}_{Q}$. This satisfies

$$
\widetilde{u}(x)=\int \sum_{Q \in \mathcal{L}} \varphi_{Q} g_{Q} d \omega^{x} \leq c|\log \varepsilon| \sum_{Q \in \mathcal{L}} \int \varphi_{Q} d \omega^{x} \leq c|\log \varepsilon|,
$$


because $\left.\sum_{Q \in \mathcal{L}} \varphi_{Q}\right|_{\partial \Omega} \leq 1$ by construction. The same arguments in the proof of Lemma 3.7 , with $u$ replaced by $\widetilde{u}$, show then that

$$
\sum_{Q \in \mathcal{L}} \mu(Q) \lesssim \varepsilon \mu(R)
$$

with the implicit constant independent of $\# \mathcal{L}$. So (3.15) holds and, also, (3.16).

\subsection{The packing condition of the family $\operatorname{Top}\left(R_{0}\right)$.}

Lemma 3.9. There exists a constant $C$ such that for any $Q_{0} \in \mathcal{D}_{\mu}\left(R_{0}\right)$,

$$
\sum_{R \in \operatorname{Top}\left(R_{0}\right): R \subset Q_{0}} \mu(R) \leq C \mu\left(Q_{0}\right) .
$$

Proof. First note that it is enough to prove the lemma assuming that $Q_{0} \in \operatorname{Top}\left(R_{0}\right)$. Indeed, given any arbitrary $Q_{0} \in \mathcal{D}_{\mu}\left(R_{0}\right)$, we consider the family $\mathcal{M}$ of maximal cubes from $\operatorname{Top}\left(R_{0}\right) \cap \mathcal{D}_{\mu}\left(R_{0}\right)$, and apply (3.23) to each $S \in \mathcal{M}$ to obtain

$$
\sum_{R \in \operatorname{Top}\left(R_{0}\right): R \subset Q_{0}} \mu(R)=\sum_{S \in \mathcal{M}} \sum_{R \in \operatorname{Top}\left(R_{0}\right): R \subset S} \mu(R) \leq C \sum_{S \in \mathcal{M}} \mu(S) \leq C \mu(R) .
$$

Therefore we assume that $Q_{0} \in \operatorname{Top}\left(R_{0}\right)$. We denote

$$
\widetilde{\operatorname{Top}}_{H}\left(Q_{0}\right):=\mathcal{D}_{\mu}\left(Q_{0}\right) \cap \operatorname{Top}_{H}\left(R_{0}\right) \quad \text { and } \quad \widetilde{\operatorname{Top}_{L}}\left(Q_{0}\right):=\mathcal{D}_{\mu}\left(Q_{0}\right) \cap \operatorname{Top}_{L}\left(R_{0}\right) \text {. }
$$

We split $\mathcal{D}_{\mu}\left(Q_{0}\right)$ into trees whose roots are all the cubes from $\left\{Q_{0}\right\} \cup \widetilde{\operatorname{Top}}_{H}\left(Q_{0}\right)$. That is, for each $R \in\left\{Q_{0}\right\} \cup{\widetilde{\operatorname{Top}_{H}}}_{H}\left(Q_{0}\right)$, we consider the tree $\widetilde{\operatorname{Tree}}(R)$ formed by the cubes from $\mathcal{D}_{\mu}(R)$ which are not contained in any other cube from $\widetilde{\operatorname{Top}}_{H}\left(Q_{0}\right) \cap \mathcal{D}_{\mu}(R)$ different from $R$. So we have the partition

$$
\mathcal{D}_{\mu}\left(Q_{0}\right)=\underset{R \in\left\{Q_{0}\right\} \cup \widetilde{\operatorname{Top}}_{H}\left(Q_{0}\right)}{ } \widetilde{\operatorname{Tree}}(R) .
$$

Also, we denote by $\operatorname{Next}(R)$ the family of the maximal cubes from $\widetilde{\operatorname{Top}}_{H}\left(Q_{0}\right) \cap \mathcal{D}_{\mu}(R)$ different from $R$.

By construction, for each $R \in\left\{Q_{0}\right\} \cup{\widetilde{\operatorname{Top}_{H}}}\left(Q_{0}\right)$ (taking into account that $Q_{0} \in$ $\left.\operatorname{Top}\left(R_{0}\right)\right)$ we have

$$
\operatorname{Next}(R) \subset \bigcup_{k \geq 0} \bigcup_{Q \in \operatorname{LD}^{k}(R)} \operatorname{HD}(Q) .
$$

Then, by Lemmas 3.4 and 3.7,

$$
\sum_{P \in \operatorname{Next}(R)} \mu(P) \leq \sum_{k \geq 0} \sum_{Q \in \mathrm{LD}^{k}(R)} \sum_{S \in \mathrm{HD}(Q)} \mu(S) \leq \frac{C}{A} \sum_{k \geq 0} \sum_{Q \in \mathrm{LD}^{k}(R)} \mu(Q) \leq \frac{C}{A} \mu(R) .
$$

So, assuming $A$ big enough, we have

$$
\sum_{P \in \operatorname{Next}(R)} \mu(P) \leq \frac{1}{2} \mu(R)
$$


which is equivalent to saying that

$$
\mu\left(R \backslash \bigcup_{P \in \operatorname{Next}(R)} P\right) \geq \frac{1}{2} \mu(R) .
$$

Since the sets $R \backslash \bigcup_{P \in \operatorname{Next}(R)} P$, with $R \in \widetilde{\operatorname{Top}}_{H}\left(Q_{0}\right)$ are pairwise disjoint, we obtain

$$
\sum_{R \in \widehat{\operatorname{Top}}_{H}\left(Q_{0}\right)} \mu(R) \leq 2 \sum_{R \in \widehat{\operatorname{Top}}_{H}\left(Q_{0}\right)} \mu\left(R \backslash \bigcup_{P \in \operatorname{Next}(R)} P\right) \leq 2 \mu\left(Q_{0}\right) .
$$

Now it remains to bound the sum $\sum_{R \in \widetilde{\operatorname{Top}}_{L}\left(Q_{0}\right)} \mu(R)$. In view of (3.24) we can split this sum as follows:

$$
\sum_{R \in \operatorname{Top}_{L}\left(Q_{0}\right)} \mu(R)=\sum_{S \in\left\{Q_{0}\right\} \cup \widetilde{\operatorname{Top}_{H}}\left(Q_{0}\right)} \sum_{R \in \widetilde{\operatorname{Tree}}(S) \cap \widetilde{\operatorname{Top}}_{L}\left(Q_{0}\right)} \mu(R) .
$$

By construction, since $Q_{0} \in \operatorname{Top}\left(R_{0}\right)$, for each $S \in\left\{Q_{0}\right\} \cup \widetilde{\operatorname{Top}}_{H}\left(Q_{0}\right)$ we have

$$
\sum_{R \in \widetilde{\operatorname{Tree}}(S) \cap{\widetilde{\operatorname{Top}_{L}}}_{L}\left(Q_{0}\right)} \mu(R) \leq \sum_{k \geq 0} \sum_{Q \in \operatorname{LD}^{k}(S)} \mu(Q),
$$

which does not exceed $C \mu(S)$ by Lemma 3.7 again, so that

$$
\sum_{R \in \overline{\operatorname{Top}}_{L}\left(Q_{0}\right)} \mu(R) \leq C \sum_{S \in\left\{Q_{0}\right\} \cup{\widetilde{\operatorname{Top}_{H}}}_{H}\left(Q_{0}\right)} \mu(S) \leq C \mu\left(Q_{0}\right),
$$

by (3.25). This completes the proof of the lemma.

3.7. Conclusion of the proof of Proposition 3.1. If $\operatorname{diam}(\partial \Omega)<\infty$, then we choose $R_{0}=\partial \Omega$ and we define Top $=\operatorname{Top}\left(R_{0}\right)$. By Lemmas 3.6 and 3.9, the family Top satisfies the properties required in Proposition 3.1.

In the case when $\partial \Omega$ is not bounded we apply a technique described in p. 38 of [DS1]: we consider a family of cubes $\left\{R_{j}\right\}_{j \in J} \in \mathcal{D}_{\mu}$ which are pairwise disjoint, whose union is all of $\operatorname{supp} \mu$, and which have the property that for each $k$ there at most $C$ cubes from $\mathcal{D}_{\mu, k}$ not contained in any cube $R_{j}$. For each $R_{j}$ we construct a family $\operatorname{Top}\left(R_{j}\right)$ analogous to $\operatorname{Top}\left(R_{0}\right)$. Then we set

$$
\operatorname{Top}=\bigcup_{j \in J} \operatorname{Top}\left(R_{j}\right) \cup \mathcal{B},
$$

where $\mathcal{B} \subset \mathcal{D}_{\mu}$ is the family of cubes which are not contained in any cube $R_{j}, j \in J$. One can easily check that the family Top satisfies all the properties from Proposition 3.1. See p. 38 of [DS1] for the construction of the family $\left\{R_{j}\right\}$ and additional details.

\section{PROOF OF THEOREM 1.1 FOR BOUNDED UNIFORM DOMAINS}

In the rest of the paper we allow all the constants $C$ and other implicit constants to depend on the parameter $\varepsilon$ from Subsection 3.2.

In this section we will complete the proof of Theorem 1.1 in the special case when $\Omega$ is a bounded uniform domain. For this type of domain the proof is simpler and more transparent than in the general case because the Harnack chain condition holds in a uniform domain 
and for that reason we think it is useful to first give the proof in this special case. If a uniform domain has AD-regular boundary (or more generally, it satisfies the CDC), then by the Harnack chain condition the harmonic measure $\omega^{p_{Q}}$ in $\Omega$ is doubling, with the doubling constant bounded above independently of the pole $p_{Q}$ (see [AiH]). Then by the theorem of Hofmann, Martell and Uriarte-Tuero in [HMU], the uniform rectifiability of $\partial \Omega$ is equivalent to the $A_{\infty}(\mu)$ property of $\omega^{p}$, for a fixed $p \in \Omega$, and we will use this criterion to prove Theorem 1.1 in the case of uniform domains.

4.1. The set $R \backslash B_{L}^{m}(R)$. In the rest of this section we assume that $\Omega$ is a bounded uniform domain with $n$-AD-regular boundary.

For a fixed $R \in \mathcal{D}_{\mu}$, we choose $m$ big enough so that

$$
\mu\left(R \backslash B_{L}^{m}(R)\right)=\mu(R)-\mu\left(B_{L}^{m}(R)\right) \geq \mu(R)-\frac{C}{m} \mu(R) \geq \frac{1}{2} \mu(R),
$$

by applying Lemma 3.7 .

Lemma 4.1. There is a function $g \in L^{\infty}\left(\left.\omega^{p_{R}}\right|_{R \backslash B_{L}^{m}(R)}\right)$ such that

$$
\left.\frac{1}{\mu(R)} \mu\right|_{R \backslash B_{L}^{m}(R)}=\left.g \omega^{p_{R}}\right|_{R \backslash B_{L}^{m}(R)},
$$

with

$$
\|g\|_{L^{\infty}\left(\left.\omega^{p} R\right|_{R \backslash B_{L}^{m}(R)}\right)} \leq C(\delta, m) .
$$

Proof. By the Lebesgue differentiation theorem, it is enough to show that, given any $x \in$ $R \backslash B_{L}^{m}(R)$, for any $Q \in \mathcal{D}_{\mu}$ such that $x \in Q \subset R$, with $\ell(Q)$ small enough,

$$
\mu(Q) \leq C(\delta, m) \omega^{p_{R}}(Q) \mu(R) .
$$

For such point $x$, there exists $0 \leq j \leq m-1$ and some cube $Q_{j} \in \mathrm{LD}^{j}(R)$ such that

$$
x \in Q_{j} \quad \text { but } \quad x \notin \bigcup_{P \in \operatorname{LD}\left(Q_{j}\right)} P .
$$

Consider now the cubes $R=Q_{0}, Q_{1}, \ldots, Q_{j}$ such that $x \in Q_{k} \in \operatorname{LD}^{k}(R)$ for $0 \leq k \leq j$. To simplify notation, we denote $p_{k}=p_{Q_{k}}$. For any $Q \in \mathcal{D}_{\mu}$ such that $Q \subset Q_{j}$, we have

$$
\frac{\omega^{p_{j}}(Q)}{\mu(Q)} \geq \delta \frac{\omega^{p_{j}}\left(Q_{j}\right)}{\mu\left(Q_{j}\right)} \approx \frac{\delta}{\mu\left(Q_{j}\right)}
$$

Analogously, by the definition of $\operatorname{LD}\left(Q_{k}\right)$ and the doubling property of $\omega^{p_{k}}$,

$$
\frac{\omega^{p_{k}}\left(Q_{k+1}\right)}{\mu\left(Q_{k+1}\right)} \approx \delta \frac{\omega^{p_{k}}\left(Q_{k}\right)}{\mu\left(Q_{k}\right)} \approx \frac{\delta}{\mu\left(Q_{k}\right)} .
$$

Notice that for any $k$, since $\Omega$ is a uniform domain with AD-regular boundary,

$$
\omega^{p_{R}}(Q) \approx \omega^{p_{j}}(Q) \omega^{p_{R}}\left(Q_{j}\right)
$$

and also

$$
\omega^{p_{R}}\left(Q_{k}\right) \approx \omega^{p_{k-1}}\left(Q_{k}\right) \omega^{p_{R}}\left(Q_{k-1}\right) .
$$

Therefore,

$$
\omega^{p_{R}}(Q) \approx \omega^{p_{j}}(Q) \omega^{p_{j-1}}\left(Q_{j}\right) \omega^{p_{j-2}}\left(Q_{j-1}\right) \ldots \omega^{p_{R}}\left(Q_{1}\right),
$$


where the implicit constant is of the form $C^{j} \leq C^{m}$. Plugging here the estimates (4.2) and (4.3) we get

$$
\omega^{p_{R}}(Q) \gtrsim_{m} \frac{\delta \mu(Q)}{\mu\left(Q_{j}\right)} \frac{\delta \mu\left(Q_{j}\right)}{\mu\left(Q_{j-1}\right)} \frac{\delta \mu\left(Q_{j-1}\right)}{\mu\left(Q_{j-2}\right)} \ldots \frac{\delta \mu\left(Q_{1}\right)}{\mu(R)}=\delta^{j+1} \frac{\mu(Q)}{\mu(R)} \gtrsim_{m} \delta^{m+1} \frac{\mu(Q)}{\mu(R)},
$$

which proves the lemma.

4.2. The $A_{\infty}(\mu)$ property of $\omega^{p}$. To show that $\omega^{p}$ satisfies the $A_{\infty}(\mu)$ property it is enough to prove that there exists some constant $\tau>0$ such that, for each $R \in \mathcal{D}_{\mu}$ and every $F \subset R$,

$$
\omega^{p}(F) \leq \tau \omega^{p}(R) \quad \Rightarrow \quad \mu(F) \leq \frac{3}{4} \mu(R) .
$$

By the martingale property of harmonic measure in uniform domains with $n$-AD-regular boundary, the left inequality in (4.4) implies that

$$
\omega^{p_{R}}(F) \approx \frac{\omega^{p_{R}}(F)}{\omega^{p_{R}}(R)} \approx \frac{\omega^{p}(F)}{\omega^{p}(R)} \leq \tau .
$$

See $[\mathrm{AiH}]$ for further details (or [MT, Theorem 1.3] for a more precise reference) regarding this martingale property of harmonic measure in uniform domains. Now we write

$$
\mu(F) \leq \mu\left(B_{L}^{m}(R)\right)+\mu\left(F \backslash B_{L}^{m}(R)\right) \leq \frac{1}{2} \mu(R)+\mu\left(F \backslash B_{L}^{m}(R)\right),
$$

taking into account (4.1). Recall also that, by Lemma 4.1,

$$
\left.\frac{1}{\mu(R)} \mu\right|_{R \backslash B_{L}^{m}(R)}=\left.g \omega^{p_{R}}\right|_{R \backslash B_{L}^{m}(R)},
$$

with $\|g\|_{L^{\infty}\left(\left.\omega^{p} R\right|_{R \backslash B_{L}^{m}(R)}\right)} \leq C(\delta, m)$. Together with (4.5) this implies that

$$
\mu\left(F \backslash B_{L}^{m}(R)\right) \leq C(\delta, m) \mu(R) \omega^{p_{R}}\left(F \backslash B_{L}^{m}(R)\right) \leq C(\delta, m) \tau \mu(R) \leq \frac{1}{4} \mu(R),
$$

if $\tau$ is assumed small enough. Plugging this into (4.6), we obtain (4.4).

\section{FROM THE CORONA DECOMPOSITION FOR HARMONIC MEASURE TO UNIFORM RECTIFIABILITY}

In this section we will show that the existence of a corona decomposition such as the one described in Proposition 3.1 implies the uniform rectifiability of $\partial \Omega$. We will prove this by showing that the Riesz transform $\mathcal{R}_{\mu}$ is bounded in $L^{2}(\mu)$, and then applying the main theorem of [NToV1].

The precise result that we will prove is the following.

Proposition 5.1. Let $\Omega \subset \mathbb{R}^{n+1}$ be a corkscrew domain with $n$-AD-regular boundary. Denote by $\mu$ the surface measure on $\partial \Omega$. Suppose that $\mu$ admits a corona decomposition $\mathcal{D}_{\mu}=\bigcup_{R \in \text { Top }} \operatorname{Tree}(R)$ so that the family Top is a Carleson family, that is,

$$
\sum_{R \subset S: R \in \mathrm{Top}} \mu(R) \leq C \mu(S) \quad \text { for all } S \in \mathcal{D}_{\mu},
$$

and for each $R \in$ Top there exists a corkscrew point $p_{R} \in \Omega$ with

$$
c^{-1} \ell(R) \leq \operatorname{dist}\left(p_{R}, R\right) \leq \operatorname{dist}\left(p_{R}, \partial \Omega\right) \leq c \ell(R)
$$


such that, for some fixed $\lambda_{0}>1$,

$$
\omega^{p_{R}}\left(\lambda_{0} Q\right) \approx \frac{\mu(Q)}{\mu(R)} \quad \text { for all } Q \in \operatorname{Tree}(R),
$$

with the implicit constant uniform on $Q$ and $R$. Then $\mu$ is uniformly rectifiable.

Note that this proposition, when combined with Proposition 3.1, completes the proof of Theorem 1.1. Note also that from a family $\mathcal{F}$ as described in Theorem 1.3 one can construct a corona decomposition like that in Proposition 5.1, with $\lambda_{0}=7$. Indeed, if we let Top be the family of children of all cubes from $\mathcal{F}$, together with $R_{0}=\partial \Omega$ if $\operatorname{diam}(\Omega)=\infty$, and then for $R \in$ Top we let $\operatorname{Tree}(R)$ be the family of cubes $Q \in \mathcal{D}_{\mu}(R)$ which are not contained in any cube from Top $\cap \mathcal{D}_{\mu}(R)$ different from $R$, then it is easy to check that the properties stated in the above proposition ${ }^{2}$ hold for this definition of Top. Hence the combination of Propositions 3.1 and 5.1 also yields Theorem 1.3.

In the rest of this section we suppose that the assumptions of Proposition 5.1 hold for the family Top $\subset \mathcal{D}_{\mu}$. Further, for simplicity we will assume that $\lambda_{0}=3$. Very minor modifications, which we leave for the reader, yield the conclusion in the case $\lambda_{0}>1$.

5.1. The Riesz transform of $\omega^{p_{R}}$. Given $R \in$ Top, we denote by $\operatorname{Stop}(R)$ the family of cubes $Q \in \operatorname{Tree}(R)$ such that their children do not belong to $\operatorname{Tree}(R)$.

The connection between the Riesz transform operator and harmonic measure is provided by the following result.

Lemma 5.2. For $x \in R$, denote

$$
\ell(x)= \begin{cases}0 & \text { if } x \in R \backslash \bigcup_{Q \in \operatorname{Stop}(R)} Q, \\ \ell(Q) & \text { if } x \in Q \in \operatorname{Stop}(R) .\end{cases}
$$

Then,

$$
\sup _{t>\ell(x)}\left|\mathcal{R}_{t} \omega^{p_{R}}(x)\right| \lesssim \frac{1}{\mu(R)} .
$$

Proof. First note that for all $t \geq 2 \ell(R)$ and $x \in R$,

$$
\left|\mathcal{R}_{t} \omega^{p_{R}}(x)\right| \leq \frac{\left\|\omega^{p_{R}}\right\|}{\ell(R)^{n}} \lesssim \frac{1}{\mu(R)} .
$$

Next we will show that for $x \in Q \in \operatorname{Tree}(R)$ and $\ell(Q) \leq t \leq 2 \ell(Q)$,

$$
\left|\mathcal{R}_{t} \omega^{p_{R}}(x)\right| \lesssim \frac{1}{\mu(R)} .
$$

Clearly, this suffices to prove the lemma. Let $p_{Q}$ be the corkscrew point associated with the cube $Q$, as defined at the beginning of Section 3.2. Now, by standard Calderón-Zygmund estimates, using that all the ancestors of $Q$ in $\operatorname{Tree}(R)$ satisfy

$$
\omega^{p_{R}}(3 Q) \lesssim \frac{\mu(Q)}{\mu(R)}
$$

\footnotetext{
${ }^{2}$ We do not choose Top $=\mathcal{F}$ because this would not guarantee the property (3) in the definition of the corona decomposition in Subsection 3.1.
} 
and that $\left\|\omega^{p_{R}}\right\|=1$ for the ancestors that do not belong to $\operatorname{Tree}(R)$, it is easy to prove that

$$
\left|\mathcal{R}_{t} \omega^{p_{R}}(x)-\mathcal{R}_{r\left(V_{Q}\right)} \omega^{p_{R}}\left(p_{Q}\right)\right| \lesssim \sup _{\lambda \geq 1} \frac{\omega^{p_{R}}(\lambda Q)}{\ell(\lambda Q)^{n}} \lesssim \frac{1}{\mu(R)} .
$$

Notice also that by the choice of $p_{Q}$,

$$
\mathcal{R}_{r\left(V_{Q}\right)} \omega^{p_{R}}\left(p_{Q}\right)=\mathcal{R} \omega^{p_{R}}\left(p_{Q}\right) .
$$

Therefore, to finish the proof of the lemma it is enough to show that

$$
\left|\mathcal{R} \omega^{p_{R}}\left(p_{Q}\right)\right| \lesssim \frac{1}{\mu(R)}
$$

From (2.5), it is clear that for all $x \in V_{Q}$ we have

$$
\left|\mathcal{R} \omega^{p_{R}}(x)\right| \lesssim \frac{1}{\ell(R)^{n}}+\left|\nabla_{x} G\left(x, p_{R}\right)\right| .
$$

Since $G\left(\cdot, p_{R}\right)$ is harmonic in $2 V_{Q}$ and positive in $\Omega$, for all $x \in V_{Q}$ we have

$$
\left|\nabla_{x} G\left(x, p_{R}\right)\right| \lesssim \frac{1}{r\left(V_{Q}\right)} \sup _{y \in 2 V_{Q}}\left|G\left(y, p_{R}\right)-G\left(p_{Q}, p_{R}\right)\right| .
$$

Then, using (2.10) and (2.11), along with the fact that $\mu$ has polynomial growth, we infer that for $y \in 2 V_{Q}$

$$
\begin{aligned}
\frac{\left|G\left(y, p_{R}\right)-G\left(p_{Q}, p_{R}\right)\right|}{r\left(V_{Q}\right)} & \lesssim \frac{\omega^{p_{R}}\left(2 \delta_{0}^{-1} Q\right)}{\ell(Q)^{n} \omega^{p_{Q}}(Q)} \\
\lesssim \delta_{0} \frac{\omega^{p_{R}}\left(2 \delta_{0}^{-1} Q\right)}{\mu\left(2 \delta_{0}^{-1} Q\right)} & \lesssim \frac{\omega^{p_{R}}(2 R)}{\mu(2 R)} \lesssim \frac{1}{\mu(R)} .
\end{aligned}
$$

Together with (5.4), this gives (5.3).

5.2. Decomposition of $\mathcal{R}_{\mu}$ in terms of the corona decomposition. Given $\eta>0$ and a function $f \in L^{2}(\mu)$, we decompose the $\eta$-truncated Riesz transform of $f \mu$ as follows. For every $Q \in \mathcal{D}_{\mu}$, we set $^{3}$

$$
\mathcal{R}_{Q}(f \mu)(x)=\chi_{Q} \int_{\max (\eta, \ell(Q) / 2)<|x-y| \leq \ell(Q)} \frac{x-y}{|x-y|^{n+1}} f(y) d \mu(y),
$$

and for every $R \in$ Top,

$$
K_{R}(f \mu)(x)=\sum_{Q \in \operatorname{Tree}(R)} \mathcal{R}_{Q}(f \mu)(x)
$$

so that

$$
\mathcal{R}_{\eta}(f \mu)=\sum_{R \in \text { Top }} K_{R}(f \mu) .
$$

Using the decomposition (5.6) we will show below that

$$
\left\|\mathcal{R}_{\eta}(f \mu)\right\|_{L^{2}\left(\left.\mu\right|_{R_{0}}\right)} \leq c\|f\|_{L^{2}(\mu)},
$$

with the constant $c$ uniform on $R_{0} \in \mathcal{D}_{\mu}$ and $\eta>0$. Clearly this yields the $L^{2}(\mu)$ boundedness of $\mathcal{R}_{\mu}$ and thus the uniform rectifiability of $\mu$, by [NToV1].

\footnotetext{
${ }^{3}$ Recall that for $Q \in \mathcal{D}_{\mu, j}$, we write $\ell(Q)=2^{-j}$ and have $\operatorname{diam}(Q) \leq \ell(Q)$.
} 
5.3. The suppressed Riesz transform. In this subsection we describe some results on singular integrals with "suppressed kernels". All the results in this subsection are due to Nazarov, Treil and Volberg [NTrV] (although we may provide different references which may be more convenient for the reader). We consider the "suppressed kernel"

$$
K_{\Phi}(x, y)=\frac{x-y}{\left(|x-y|^{2}+\Phi(x) \Phi(y)\right)^{(n+1) / 2}},
$$

which satisfies

$$
\left|K_{\Phi}(x, y)\right| \lesssim \min \left(\frac{1}{|x-y|^{n}}, \frac{1}{\Phi(x)^{n}}, \frac{1}{\Phi(y)^{n}}\right)
$$

and

$$
\left|K_{\Phi}(x, y)-K_{\Phi}\left(x^{\prime}, y\right)\right| \lesssim \frac{\left|x-x^{\prime}\right|}{|x-y|^{n+1}}, \quad \text { if } 2\left|x-x^{\prime}\right| \leq|x-y| .
$$

Since $K_{\Phi}$ is anti-symmetric an analogous estimate holds in the $y$-variable. For a proof of the above estimates see e.g. Lemma 8.2 in [Vo]. We define the associated Riesz transform by

$$
\mathcal{R}_{\Phi} \nu(x):=\int K_{\Phi}(x, y) d \nu,
$$

for a Radon measure $\nu$. We also set $\mathcal{R}_{\nu, \Phi} f:=\mathcal{R}_{\Phi}(f \nu)$, for $f \in L_{l o c}^{1}(\nu)$.

We shall now record some auxiliary results which we will use repeatedly. If $\nu$ is a positive and finite Borel measure, we define

$$
\rho_{\nu}(x):=\sup \left\{r>0: \nu\left(B(x, r)>C_{0} r^{n}\right\}\right.
$$

and set $\rho_{\nu}(x)=0$, if the set on the right hand-side is empty. From now on we assume that $\Phi: \mathbb{R}^{n+1} \rightarrow[0, \infty)$ is a 1-Lipschitz function such that $\Phi(x) \geq \rho_{\nu}(x)$ for any $x \in \mathbb{R}^{n+1}$.

Lemma 5.3 (Lemma 5.4, [To3]). If $x \in \mathbb{R}^{n+1}$ and $\varepsilon>\Phi(x)$, then

$$
\left|\mathcal{R}_{\Phi, \varepsilon} \nu(x)-\mathcal{R}_{\varepsilon} \nu(x)\right| \lesssim \sup _{r>\varepsilon} \frac{\nu(B(x, r))}{r^{n}} .
$$

Lemma 5.4 (Lemma 5.26, [To3]). Suppose that the operator $\mathcal{R}_{\nu, \Phi}$ is bounded from $L^{1}(\nu)$ to $L^{1, \infty}(\nu)$. If $s \in(0,1]$, for any $f \in L^{2}(\nu)$ it holds that

$$
\mathcal{R}_{\nu, \Phi, *} f(x) \lesssim M_{\nu}\left[\left(\mathcal{R}_{\nu, \Phi} f\right)^{s}\right](x)^{1 / s}+\left(1+\left\|\mathcal{R}_{\Phi}\right\|_{L^{1}(\nu) \rightarrow L^{1, \infty}(\nu)}\right) M_{\nu} f(x) .
$$

We remark here that in [To3] Lemma 5.4 is stated only for $s=1$. However, the same arguments (with minor adjustments) show this more general version as well.

Given $b>1$, we introduce the following suppressed Hardy-Littlewood maximal operators:

$$
\begin{aligned}
M_{\nu, b, \Phi} f(x) & :=\sup _{r \geq \Phi(x)} \frac{1}{\nu(B(x, b r))} \int_{B(x, b r)}|f(y)| d \nu(y), \\
M_{\nu, \Phi}^{r} f(x) & :=\sup _{r \geq \Phi(x)} \frac{1}{r^{n}} \int_{B(x, b r)}|f(y)| d \nu(y) .
\end{aligned}
$$

We will need the following variant of Lemma 5.4. The proof follows by inspection from the proof of Lemma 5.4 and we leave the details to the reader. 
Lemma 5.5. Suppose that the operator $\mathcal{R}_{\nu, \Phi}$ is bounded from $L^{1}(\nu)$ to $L^{1, \infty}(\nu)$. If $s \in$ $(0,1]$, then for any $f \in L^{2}(\nu)$,

$\mathcal{R}_{\Phi, *}(f \nu)(x) \lesssim M_{\nu, b, \Phi}\left(\mathcal{R}_{\Phi}(f \nu)^{s}\right)(x)^{1 / s}+\left(1+\left\|\mathcal{R}_{\Phi}\right\|_{L^{1}(\nu) \rightarrow L^{1, \infty}(\nu)}\right) M_{\nu, \Phi}^{r} f(x)+M_{\nu, b, \Phi} f(x)$, with the implicit constant depending on $s$ and $b$.

Lemma 5.6 (Lemma 5.27, [To3]). If the operator $\mathcal{R}_{\nu, \Phi}$ is bounded in $L^{2}(\nu)$, then it is also bounded from $L^{1}(\nu)$ to $L^{1, \infty}(\nu)$. Moreover,

$$
\left\|\mathcal{R}_{\nu, \Phi}\right\|_{L^{1}(\nu) \rightarrow L^{1, \infty}(\nu)} \lesssim 1+\left\|\mathcal{R}_{\nu, \Phi}\right\|_{L^{2}(\nu) \rightarrow L^{2}(\nu)} .
$$

Theorem 5.7. If there exists a constant $C_{1}>0$ such that $\mathcal{R}_{\Phi, *} \nu(x) \leq C_{1}$ for $\nu$-a.e. $x$, then $\mathcal{R}_{\nu, \Phi}$ is bounded in $L^{2}(\nu)$.

Proof. This follows from an application of the $T b$ theorem for suppressed operators of Nazarov, Treil and Volberg (see Theorem 12.1 in [Vo]). Indeed, if our test function is $b=1$, then it is always accretive and thus, the set $T_{12}$ in Theorem 12.1 is just the empty set. Therefore, since

$$
\left|K_{\Phi}(x, y)\right| \lesssim \min \left(\frac{1}{|x-y|^{n}}, \frac{1}{\Phi(x)^{n}}, \frac{1}{\Phi(y)^{n}}\right),
$$

$\Phi(x) \geq \rho_{\nu}(x)$ and, by assumption, $\mathcal{R}_{\nu, \Phi, *} \leq C_{1}$, we can apply Theorem 12.1 in [Vo] to obtain that $\mathcal{R}_{\Phi}$ is bounded in $L^{2}(\nu)$.

5.4. The $L^{p}(\mu)$-boundedness of $K_{R}(\cdot \mu)$. Our next objective consists in proving the following:

Lemma 5.8. Let $1<p<\infty$ and $f \in L^{p}(\mu)$. Then for every $R \in$ Top we have

$$
\left\|K_{R}(f \mu)\right\|_{L^{p}(\mu)} \lesssim\|f\|_{L^{p}(\mu)},
$$

with the implicit constant uniform on $R$.

We need to consider the following auxiliary function:

$$
\Phi(x)=\inf _{Q \in \operatorname{Tree}(R)}(\operatorname{dist}(x, Q)+\ell(Q)), \quad x \in \mathbb{R}^{n+1} .
$$

Notice that $\Phi$ is 1-Lipschitz. Next we define a "regularized" family Reg of cubes. For each $x \in \operatorname{supp} \mu$ such that $\Phi(x) \neq 0$, let $Q_{x}$ be a dyadic cube from $\mathcal{D}_{\mu}$ containing $x$ such that

$$
\frac{\Phi(x)}{2}<\ell\left(Q_{x}\right) \leq \Phi(x) .
$$

If $\Phi(x)=0$, we set $Q_{x}=\{x\}$. Then, $\operatorname{Reg}(R)$ is a maximal (and thus disjoint) subfamily of $\left\{Q_{x}\right\}_{x \in 3 R, \Phi(x)>0}$. Note that not all the cubes from $\operatorname{Reg}(R)$ are contained in $R$.

Lemma 5.9. The family $\operatorname{Reg}(R)$ satisfies:

(a) $\bigcup_{Q \in \operatorname{Reg}(R) \cap \mathcal{D}_{\mu}(R)} Q \subset \bigcup_{Q \in \operatorname{Stop}(R)} Q$.

(b) If $P, Q \in \operatorname{Reg}(R)$ and $2 P \cap 2 Q \neq \varnothing$, then $\ell(Q) / 2 \leq \ell(P) \leq 2 \ell(Q)$. 
(c) If $Q \in \operatorname{Reg}(R), Q \cap 3 R \neq \varnothing$, and $x \in Q, r \geq \ell(Q)$, then

$$
\omega^{p_{R}}(B(x, r)) \lesssim r^{n} / \mu(R) .
$$

(d) For each $Q \in \operatorname{Reg}(R)$ with $Q \cap 3 R \neq \varnothing$, there exists some cube $\widetilde{Q} \in \operatorname{Tree}(R)$ such that

$$
\ell(\widetilde{Q}) \approx \ell(Q) \quad \text { and } \quad \operatorname{dist}(Q, \widetilde{Q}) \lesssim \ell(Q) .
$$

The proof of this lemma follows by standard arguments (see e.g. Theorem 8.2 in [To1] or Lemma 3 in [G-S]).

We remark, that abusing notation, we may also think of the points $x \in \mathbb{R}^{d}$ such that $\Phi(x)=0$ as cubes with side length 0 . Then, if we enlarge the family $\operatorname{Reg}(R)$ by adding these cubes consisting of a single point, the resulting family, call it $\widehat{\operatorname{Reg}}(R)$, also satisfies the properties in the preceding lemma.

By the properties in Lemma 5.9 and an easy application of the Lebesgue differentiation theorem, one can see that, module a set of zero $\mu$ and $\omega^{p_{R}}$ measure.

$$
R \backslash \bigcup_{Q \in \operatorname{Stop}(R)} Q=\operatorname{supp} \mu \backslash \bigcup_{Q \in \operatorname{Reg}(R)} Q
$$

From now on, in this subsection we denote by $\widetilde{\mu}$ and $\sigma$ the measures

$$
\widetilde{\mu}=\left.\mu\right|_{\bigcup_{Q \in \operatorname{Reg}(R)}} 3 R \cap Q, \quad \sigma=\left.\mu(R) \omega^{p_{R}}\right|_{3 R} .
$$

Lemma 5.10. For all $x \in 3 R$, we have

$$
\sigma(B(x, r)) \leq C r^{n} \quad \text { for all } r \geq \Phi(x) .
$$

Proof. By the definition of $\Phi(x)$, there exists some $Q \in \operatorname{Tree}(R)$ such that

$$
\Phi(x) \leq 2(\operatorname{dist}(x, Q)+\ell(Q)) .
$$

Hence there exists some cube $S \in \mathcal{D}_{\mu}$ such that $S \supset Q$ and $3 S \supset B(x, r)$ with $\ell(S) \approx r$. In particular, either $S \in \operatorname{Tree}(R)$ or $S \supset R$ and since

we have

$$
\omega^{p_{R}}(3 S) \lesssim \frac{\mu(S)}{\mu(R)}
$$

$$
\sigma(B(x, r)) \leq \sigma(3 S)=\mu(R) \omega^{p_{R}}(3 S \cap 3 R) \lesssim \mu(S) \approx \ell(S)^{n} \approx r^{n} .
$$

From the last lemma it follows that

$$
\Phi(x) \geq \rho_{\sigma}(x)
$$

if we choose the constant $C_{0}$ in the definition (5.10) of $\rho_{\sigma}$ to be the constant $C$ on the right hand side of (5.12).

Lemma 5.11. In $3 R \backslash \bigcup_{Q \in \operatorname{Reg}(R)} Q$, we have $d \sigma(x)=h(x) d \mu(x)$, with $h(x) \approx 1$.

Proof. By the Lebesgue differentiation theorem, it follows that $\omega^{p_{R}} \ll \mu$ in $R \backslash \bigcup_{Q \in \operatorname{Stop}(R)} Q$, with $d \omega^{p_{R}}=h(x) \frac{1}{\mu(R)} \mu$ and $h(x) \approx 1$ on this set, which yields the lemma. 
Lemma 5.12. If $Q \in \operatorname{Reg}(R)$ and $x \in Q, \ell(Q) \leq r \leq 100$ diam(3R), then there exists some constant $b \geq 1$ such that

$$
\mu(B(x, r)) \lesssim \omega^{p_{R}}(B(x, b r) \cap 3 R) \mu(R) .
$$

The bounds on the constant $b$ only depend on the parameters of the construction of $\mathcal{D}_{\mu}$.

Proof. Given $x \in Q$, there exist $Q^{\prime} \in \operatorname{Tree}(R)$ and $x_{Q^{\prime}} \in Q^{\prime}$ such that

$$
\ell(Q) \leq\left|x-x_{Q^{\prime}}\right|+\ell\left(Q^{\prime}\right) \leq 2 \ell(Q) .
$$

From the above inequalities it is clear that $\left|x-x_{Q^{\prime}}\right| \leq 2 r$ and $\ell\left(Q^{\prime}\right) / 2 \leq r$. Therefore there are two cases:

Case (i): There exists $Q^{\prime \prime} \in \operatorname{Tree}(R)$ such that $B(x, r) \cap \operatorname{supp} \mu \subset 3 Q^{\prime \prime}$ and $\ell\left(Q^{\prime \prime}\right) \approx r$. Trivially, one can find $b \approx 1$ such that $Q^{\prime \prime} \subset B(x, b r) \cap 3 R$. Therefore,

$$
\mu(B(x, r)) \leq \mu\left(3 Q^{\prime \prime}\right) \lesssim \mu\left(Q^{\prime \prime}\right) \lesssim \omega^{p_{R}}\left(3 Q^{\prime \prime}\right) \mu(R) \leq \omega^{p_{R}}(B(x, b r) \cap 3 R) \mu(R),
$$

where in the penultimate inequality we used that $\omega^{p_{R}}\left(3 Q^{\prime \prime}\right) \approx \frac{\mu\left(Q^{\prime \prime}\right)}{\mu(R)}$.

Case (ii): $3 R \subset B(x, r)$. Since $r \leq 100 \operatorname{diam}(3 R)$, for some $C>1$,

$$
\mu(B(x, r)) \leq \mu(C R) \lesssim \omega^{p_{R}}(R) \mu(R) \leq \omega^{p_{R}}(B(x, r) \cap 3 R) \mu(R),
$$

which concludes our lemma.

Lemma 5.13. The operators $M_{\sigma, b, \Phi}$ and $M_{\sigma, \Phi}^{r}$ are bounded from $L^{p}(\sigma)$ to $L^{p}(\widetilde{\mu}), 1<p \leq$ $\infty$, and from $L^{1}(\sigma)$ to $L^{1, \infty}(\widetilde{\mu})$, with norms depending $p$.

Proof. The boundedness of $M_{\sigma, \Phi}^{r}$ is well known; see for instance in the proof of Lemma 7.6 in [To2]. Concerning $M_{\sigma, b, \Phi}$, it is enough to show that this is bounded from $L^{1}(\sigma)$ to $L^{1, \infty}(\widetilde{\mu})$, since this is trivially bounded from $L^{\infty}(\sigma)$ to $L^{\infty}(\widetilde{\mu})$. To this end, let $f \in L^{1}(\sigma)$ and for fixed $\lambda>0$ set

$$
\Omega_{\lambda}:=\left\{x \in \bigcup_{Q \in \operatorname{Reg}(R)} 3 R \cap Q: M_{\sigma, b, \Phi} f(x)>\lambda\right\} .
$$

By definition, for each $x \in \Omega_{\lambda}$, there exists a ball $B_{x}$ centered at $x$, with radius $r\left(B_{x}\right) \geq$ $\Phi(x)$ such that

$$
\frac{1}{\lambda} \int_{b B_{x}}|f(y)| d \sigma(y)>\sigma\left(b B_{x}\right)=\omega^{p_{R}}\left(b B_{x} \cap 3 R\right) \mu(R) .
$$

Further, we may assume that $r\left(B_{x}\right) \leq \operatorname{diam}(3 R)$ because $\operatorname{supp} \sigma \cup \operatorname{supp} \widetilde{\mu} \subset \overline{3 R}$. By Vitali's $5 r$-covering lemma, we can find a countable family of balls $\left\{B_{i}\right\}$ such that $b B_{i} \cap$ $b B_{j}=\varnothing$ for $i \neq j$, and

$$
\bigcup_{x \in \Omega_{\lambda}} B_{x} \subset \bigcup_{i} 5 b B_{i}
$$

Since each ball $B_{i}$ is centered at some point $x \in Q \in \operatorname{Reg}(R)$, by Lemma 5.12 we have that

$$
\mu\left(B_{i}\right) \leq \omega^{p_{R}}\left(b B_{i} \cap 3 R\right) \mu(R) .
$$


Then we deduce

$$
\begin{aligned}
\widetilde{\mu}\left(\Omega_{\lambda}\right) & \leq \sum_{i} \widetilde{\mu}\left(5 b B_{i}\right) \lesssim_{b} \sum_{i} \mu\left(B_{i}\right) \lesssim \sum_{i} \omega^{p_{R}}\left(b B_{i} \cap 3 R\right) \mu(R) \\
& \leq \sum_{i} \frac{1}{\lambda} \int_{b B_{i}}|f(y)| d \sigma(y) \leq \frac{\|f\|_{L^{1}(\sigma)}}{\lambda}
\end{aligned}
$$

which finishes our proof.

Lemma 5.14. The operator $\mathcal{R}_{\sigma, \Phi}$ is bounded in $L^{p}(\sigma), 1<p<\infty$, and from $L^{1}(\sigma)$ to $L^{1, \infty}(\sigma)$, with norm depending on $p$.

Proof. We first prove that $\mathcal{R}_{\sigma, \Phi}$ is bounded in $L^{p}(\sigma)$ for $1<p<\infty$. Taking into account Lemma 5.10, by Theorem 5.7, it is enough to show that

$$
\mathcal{R}_{\Phi, *} \sigma(x) \lesssim 1 \quad \text { for all } x \in 3 R .
$$

For any $x \in 3 R$, we write

$$
\mathcal{R}_{\Phi, *}\left[\chi_{3 R} \omega^{p_{R}}\right](x) \leq \mathcal{R}_{\Phi, *} \omega^{p_{R}}(x)+\mathcal{R}_{\Phi, *}\left[\chi_{(3 R)^{c}} \omega^{p_{R}}\right](x) .
$$

Let us estimate the first term on the right hand side. Suppose first that $x \in Q \in \operatorname{Reg}(R)$, with $Q \cap 3 R \neq \varnothing$. By Lemma 5.9 (d), there exists some cube $\widetilde{Q} \in \operatorname{Tree}(R)$ such that

$$
\ell(\widetilde{Q}) \approx \ell(Q) \text { and } \operatorname{dist}(Q, \widetilde{Q}) \lesssim \ell(Q) .
$$

By Lemma 5.2, it holds that for any $\widetilde{x} \in \widetilde{Q}$,

$$
\sup _{t>\ell(\widetilde{Q})}\left|\mathcal{R}_{t} \omega^{p_{R}}(\widetilde{x})\right| \lesssim \frac{1}{\mu(R)}
$$

By standard estimates, it follows that

$$
\sup _{t>\ell(Q)}\left|\mathcal{R}_{t} \omega^{p_{R}}(x)\right| \lesssim \sup _{t>\ell(\widetilde{Q})}\left|\mathcal{R}_{t} \omega^{p_{R}}(\widetilde{x})\right|+\sup _{t>\ell(\widetilde{Q})} \frac{\omega^{p_{R}}(B(\widetilde{x}, t))}{t^{n}} .
$$

From the properties of the cubes from $\operatorname{Tree}(R)$, it follows easily that

$$
\sup _{t>\ell(\widetilde{Q})} \frac{\omega^{p_{R}}(B(\widetilde{x}, t))}{t^{n}} \lesssim \frac{1}{\mu(R)}
$$

Together with (5.16), this gives

$$
\sup _{t>\ell(Q)}\left|\mathcal{R}_{t} \omega^{p_{R}}(x)\right| \lesssim \frac{1}{\mu(R)}
$$

This, in turn, implies that if $x \in \bigcup_{Q \in \operatorname{Reg}(R)} Q$,

$$
\mathcal{R}_{\Phi, *} \omega^{p_{R}}(x) \lesssim \frac{1}{\mu(R)}
$$

Indeed, notice that if $x \in Q \in \operatorname{Reg}(R)$ and $\varepsilon \leq 2 \Phi(x)$, then by standard estimates, we have that

$$
\left|\mathcal{R}_{\Phi, \varepsilon} \omega^{p_{R}}(x)-\mathcal{R}_{\Phi, 2 \Phi(x)} \omega^{p_{R}}(x)\right| \lesssim \sup _{r>2 \Phi(x)} \frac{\omega^{p_{R}}(B(x, r))}{r^{n}} \leq \sup _{r>\ell(Q)} \frac{\omega^{p_{R}}(B(x, r))}{r^{n}} \lesssim \frac{1}{\mu(R)},
$$


where in the penultimate inequality we used that $\Phi(x) \geq \ell(Q) / 2$ and in the last one we used Lemma 5.9 (c). Moreover, by Lemma 5.3 and similar considerations,

$$
\left|\mathcal{R}_{2 \Phi(x)} \omega^{p_{R}}(x)-\mathcal{R}_{\Phi, 2 \Phi(x)} \omega^{p_{R}}(x)\right| \lesssim \sup _{r>2 \Phi(x)} \frac{\omega^{p_{R}}(B(x, r))}{r^{n}} \lesssim \frac{1}{\mu(R)} .
$$

The latter two estimates combined with (5.17) imply that for $\varepsilon \leq 2 \Phi(x)$,

$$
\left|\mathcal{R}_{\Phi, \varepsilon} \omega^{p_{R}}(x)\right| \lesssim \frac{1}{\mu(R)}+\left|\mathcal{R}_{2 \Phi(x)} \omega^{p_{R}}(x)\right| \lesssim \frac{1}{\mu(R)} .
$$

On the other hand, in view of (5.17), it is clear that

$$
\sup _{\varepsilon>2 \Phi(x)}\left|\mathcal{R}_{\Phi, \varepsilon} \omega^{p_{R}}(x)\right| \leq \sup _{\varepsilon>\ell(Q)}\left|\mathcal{R}_{\Phi, \varepsilon} \omega^{p_{R}}(x)\right| \lesssim \frac{1}{\mu(R)},
$$

which concludes (5.18).

In the case $x \in 3 R \backslash \bigcup_{Q \in \operatorname{Reg}(R)} Q$, we have $\Phi(x)=0$ and a direct application of Lemma 5.2 shows that (5.18) also holds.

Next we estimate the last term in (5.15). To this end, note that $\Phi(y) \gtrsim \ell(R)$ for all $y \in(3 R)^{c}$. Hence, for $x \in 3 R$ and $y \in(3 R)^{c}$, we have

So we get

$$
\left|K_{\Phi}(x, y)\right| \lesssim \frac{1}{\Phi(y)^{n}} \lesssim \frac{1}{\ell(R)^{n}}
$$

In combination with (5.18), this gives

$$
\mathcal{R}_{\Phi, *}\left[\chi_{(3 R)^{c}} \omega^{p_{R}}\right](x) \lesssim \frac{\omega^{p_{R}}\left((3 R)^{c}\right)}{\ell(R)^{n}} \leq \frac{1}{\ell(R)^{n}} \lesssim \frac{1}{\mu(R)} .
$$

$$
\mathcal{R}_{\Phi, *}\left[\chi_{3 R} \omega^{p_{R}}\right](x) \lesssim \frac{1}{\mu(R)} \quad \text { for all } x \in 3 R .
$$

This finishes the proof of (5.14) and of the $L^{2}(\sigma)$ boundedness of $\mathcal{R}_{\sigma, \Phi}$. Together with Lemma 5.6, this implies that $\mathcal{R}_{\sigma, \Phi}$ is bounded from $L^{1}(\sigma)$ to $L^{1, \infty}(\sigma)$, and thus in $L^{p}(\sigma)$ for $1<p<\infty$. Our lemma is now concluded.

Lemma 5.15. The operator $\mathcal{R}_{\sigma, \Phi}$ is bounded from $L^{p}(\sigma)$ to $L^{p}(\widetilde{\mu}), 1<p<\infty$, and from $L^{1}(\sigma)$ to $L^{1, \infty}(\widetilde{\mu})$, with the norms depending on $p$.

Proof. Note that Lemma 5.5 holds for $\nu=\sigma$. Then that $\mathcal{R}_{\sigma, \Phi}$ is bounded from $L^{p}(\sigma)$ to $L^{p}(\widetilde{\mu})$ follows by a direct application of Lemma 5.13 and Lemma 5.14. The same lemmas also imply that $\mathcal{R}_{\sigma, \Phi}$ is bounded from $L^{1}(\sigma)$ to $L^{1, \infty}(\widetilde{\mu})$ in a non-trivial way. Although the arguments are standard, we will give the proof for the sake of clarity.

In view of Cotlar's inequality in Lemma 5.5 and Lemma 5.13, it suffices to prove that for $f \in L^{1}(\sigma)$ and $\lambda>0$ it holds that

$$
\widetilde{\mu}\left(\left\{\left(M_{\sigma, b, \Phi}\left(\mathcal{R}_{\Phi, \sigma} f^{s}\right)\right)^{1 / s}>\lambda\right\}\right) \lesssim \lambda^{-1}\|f\|_{L^{1}(\sigma)} .
$$

Define now

$$
g:=\left|\mathcal{R}_{\sigma, \Phi} f\right|^{s}, \quad g_{1}:=g \chi_{\left\{|g|<\lambda^{s} / 2\right\}} \quad \text { and } \quad g_{2}:=g-g_{1} .
$$

Set also $E_{\lambda}:=\left\{\left|\mathcal{R}_{\sigma, \Phi} f\right|>\frac{\lambda}{2^{1 / s}}\right\}$. Since

$$
\widetilde{\mu}\left(\left\{\left(M_{\sigma, b, \Phi} g\right)^{1 / s}>\lambda\right\}\right) \leq \widetilde{\mu}\left(\left\{M_{\sigma, b, \Phi} g_{1}>\lambda^{s} / 2\right\}\right)+\widetilde{\mu}\left(\left\{M_{\sigma, b, \Phi} g_{2}>\lambda^{s} / 2\right\}\right)
$$


and $M_{\sigma, b, \Phi} g_{1} \leq \lambda^{s} / 2$, it is enough to prove that

$$
\widetilde{\mu}\left(\left\{M_{\sigma, b, \Phi} g_{2}>\lambda^{s} / 2\right\}\right) \lesssim\|f\|_{L^{1}(\sigma)} .
$$

To this end, in light of Lemma 5.13, Kolmogorov's inequality (see e.g. Lemma 2.19 in [To3]) and Lemma 5.14 (i.e. $\mathcal{R}_{\sigma, \Phi}$ is bounded from $L^{1}(\sigma)$ to $L^{1, \infty}(\sigma)$ ), we get that

$$
\begin{aligned}
\widetilde{\mu}\left(\left\{M_{\sigma, b, \Phi} g_{2}>\lambda^{s} / 2\right\}\right) & \lesssim \lambda^{-s} \int\left|g_{2}\right| d \sigma=\lambda^{-s} \int_{E_{\lambda}}\left|\mathcal{R}_{\sigma, \Phi} f\right|^{s} d \sigma \\
& \lesssim \lambda^{-s} \sigma\left(E_{\lambda}\right)^{1-s}\left\|\mathcal{R}_{\sigma, \Phi} f\right\|_{L^{1, \infty}(\sigma)}^{s} \\
& \lesssim \lambda^{-s} \lambda^{s-1}\|f\|_{L^{1}(\sigma)}^{1-s}\|f\|_{L^{1}(\sigma)}^{s} \\
& =\lambda^{-1}\|f\|_{L^{1}(\sigma)} .
\end{aligned}
$$

This finishes the proof of the lemma.

Remark 5.16. Since $K_{\Phi}$ is antisymmetric, by duality, $\mathcal{R}_{\widetilde{\mu}, \Phi}: L^{p}(\widetilde{\mu}) \rightarrow L^{p}(\sigma)$ is bounded, for $1<p<\infty$.

Next we intend to show that $\mathcal{R}_{\widetilde{\mu}, \Phi}$ is bounded in $L^{p}(\widetilde{\mu})$.

Lemma 5.17. The operator $\mathcal{R}_{\widetilde{\mu}, \Phi}$ is bounded in $L^{p}(\widetilde{\mu})$, for $1<p<\infty$, with its norm depending on $p$.

Proof. It is enough to prove that $\mathcal{R}_{\widetilde{\mu}, \Phi}$ is bounded from $L^{1}(\widetilde{\mu})$ to $L^{1, \infty}(\widetilde{\mu})$ because as shown in [To2, Proposition 7.8], for example, this implies the $L^{p}(\mu)$-boundedness for $1<p<\infty$. To this end, let $f \in L^{1}(\widetilde{\mu})$ and for each $Q \in \operatorname{Reg}(R)$ such that $Q \cap 3 R \neq \varnothing$, consider the function $\varphi_{Q}$ defined by

$$
\varphi_{Q}=\chi_{a Q} \frac{1}{\sigma(a Q)} \int_{Q} f d \widetilde{\mu}
$$

where $a>1$ will be fixed in a moment. In this way, we have

$$
\left\|\varphi_{Q}\right\|_{L^{1}(\sigma)}=\int_{a Q} \varphi_{Q} d \sigma=\int_{Q} f d \widetilde{\mu} .
$$

Further, if $a$ is chosen big enough (i.e., $a \gtrsim b$ ), by Lemma 5.12 we have $\sigma(a Q) \gtrsim \mu(Q)$, and so

$$
\left\|\varphi_{Q}\right\|_{L^{2}(\sigma)} \leq \frac{1}{\sigma(a Q)^{1 / 2}} \int_{Q} f d \widetilde{\mu} \lesssim \frac{1}{\mu(Q)^{1 / 2}} \int_{Q} f d \widetilde{\mu} .
$$

Now we we write

$$
f \widetilde{\mu}=\sum_{Q \in \operatorname{Reg}(R)}\left(\left.f \widetilde{\mu}\right|_{Q}-\varphi_{Q} \sigma\right)+\sum_{Q \in \operatorname{Reg}(R)} \varphi_{Q} \sigma=: \nu+\eta,
$$

which implies that

$$
\widetilde{\mu}\left(\left\{x:\left|\mathcal{R}_{\widetilde{\mu}, \Phi} f(x)\right|>\lambda\right\}\right) \leq \widetilde{\mu}\left(\left\{x:\left|\mathcal{R}_{\Phi} \nu(x)\right|>\lambda / 2\right\}\right)+\widetilde{\mu}\left(\left\{x:\left|\mathcal{R}_{\Phi} \eta(x)\right|>\lambda / 2\right\}\right) .
$$

To deal with the last term above, we use the boundedness of $\mathcal{R}_{\sigma, \Phi}$ from $L^{1}(\sigma)$ to $L^{1, \infty}(\widetilde{\mu})$, proved in Lemma 5.15:

$$
\widetilde{\mu}\left(\left\{x:\left|\mathcal{R}_{\Phi} \eta(x)\right|>\lambda / 2\right\}\right) \leq C \frac{\left\|\sum_{Q \in \operatorname{Reg}(R)} \varphi_{Q}\right\|_{L^{1}(\sigma)}}{\lambda} .
$$


Observe now that

$$
\sum_{Q \in \operatorname{Reg}(R)}\left\|\varphi_{Q}\right\|_{L^{1}(\sigma)} \leq \sum_{Q \in \operatorname{Reg}(R)} \int_{Q}|f| d \widetilde{\mu}=\|f\|_{L^{1}(\widetilde{\mu})}
$$

Hence,

$$
\widetilde{\mu}\left(\left\{x:\left|\mathcal{R}_{\Phi} \eta(x)\right|>\lambda / 2\right\}\right) \leq C \frac{\|f\|_{L^{1}(\widetilde{\mu})}}{\lambda} .
$$

To estimate the first term on the right hand side of (5.20), we set

$$
\widetilde{\mu}\left(\left\{x:\left|\mathcal{R}_{\Phi} \nu(x)\right|>\lambda / 2\right\}\right) \leq \frac{1}{\lambda} \int\left|\mathcal{R}_{\Phi} \nu\right| d \widetilde{\mu} \leq \frac{1}{\lambda} \sum_{Q \in \operatorname{Reg}(R)} \int\left|\mathcal{R}_{\Phi} \nu_{Q}\right| d \widetilde{\mu}
$$

where we wrote

$$
\nu_{Q}=\left.f \widetilde{\mu}\right|_{Q}-\varphi_{Q} \sigma
$$

Now we split

$$
\begin{aligned}
\int\left|\mathcal{R}_{\Phi} \nu_{Q}\right| d \widetilde{\mu} & =\int_{2 a Q}\left|\mathcal{R}_{\Phi} \nu_{Q}\right| d \widetilde{\mu}+\int_{(2 a Q)^{c}}\left|\mathcal{R}_{\Phi} \nu_{Q}\right| d \widetilde{\mu} \\
& \leq \int_{2 a Q}\left|\mathcal{R}_{\Phi}\left(\left.f \widetilde{\mu}\right|_{Q}\right)\right| d \widetilde{\mu}+\int_{2 a Q}\left|\mathcal{R}_{\Phi}\left(\varphi_{Q} \sigma\right)\right| d \widetilde{\mu}+\int_{(2 a Q)^{c}}\left|\mathcal{R}_{\Phi} \nu_{Q}\right| d \widetilde{\mu} .
\end{aligned}
$$

For the first summand on the right hand side, using that $\Phi(x) \approx \ell(Q)$ for all $x \in Q$, we get

$$
\begin{aligned}
\int_{2 a Q}\left|\mathcal{R}_{\Phi}\left(\left.f \widetilde{\mu}\right|_{Q}\right)\right| d \widetilde{\mu} & \leq \mu(2 a Q)\left\|\mathcal{R}_{\Phi}\left(\left.f \widetilde{\mu}\right|_{Q}\right)\right\|_{L^{\infty}(\widetilde{\mu})} \\
& \lesssim \mu(2 a Q) \frac{\left\|\chi_{Q} f\right\|_{L^{1}(\widetilde{\mu})}}{\ell(Q)^{n}} \lesssim\left\|\chi_{Q} f\right\|_{L^{1}(\widetilde{\mu})} .
\end{aligned}
$$

For the second summand on the right hand side of (5.21) we use that $\mathcal{R}_{\sigma, \Phi}$ is bounded from $L^{2}(\sigma)$ to $L^{2}(\widetilde{\mu})$, by Lemma 5.15:

$$
\int_{2 a Q}\left|\mathcal{R}_{\Phi}\left(\varphi_{Q} \sigma\right)\right| d \widetilde{\mu} \leq \widetilde{\mu}(2 a Q)^{1 / 2}\left\|\mathcal{R}_{\Phi}\left(\varphi_{Q} \sigma\right)\right\|_{L^{2}(\widetilde{\mu})} \lesssim \mu(Q)^{1 / 2}\left\|\varphi_{Q}\right\|_{L^{2}(\sigma)}
$$

Using the estimate (5.19) for $\left\|\varphi_{Q}\right\|_{L^{2}(\sigma)}$, we derive

$$
\int_{2 a Q}\left|\mathcal{R}_{\Phi}\left(\varphi_{Q} \sigma\right)\right| d \widetilde{\mu} \lesssim\left\|\chi_{Q} f\right\|_{L^{1}(\widetilde{\mu})}
$$

To bound the last integral in (5.21), we take into account that $\int d \nu_{Q}=0$, and so for all $x \notin 2 a Q$,

$$
\left|\mathcal{R}_{\Phi} \nu_{Q}(x)\right| \leq \int\left|K_{\Phi}(x, y)-K_{\Phi}\left(x, z_{Q}\right)\right| d\left|\nu_{Q}\right|(y)
$$

where $z_{Q}$ denotes the center of $Q$. Since $\left|K_{\Phi}(x, y)-K_{\Phi}\left(x, z_{Q}\right)\right| \lesssim \ell(Q) /\left|x-z_{Q}\right|^{n+1}$, we derive

and thus

$$
\left|\mathcal{R}_{\Phi} \nu_{Q}(x)\right| \lesssim \frac{\left\|\nu_{Q}\right\|}{\left|x-z_{Q}\right|^{n+1}}
$$

$$
\int_{(2 a Q)^{c}}\left|\mathcal{R}_{\Phi} \nu_{Q}\right| d \widetilde{\mu} \lesssim\left\|\nu_{Q}\right\| \int_{(2 a Q)^{c}} \frac{1}{\left|x-z_{Q}\right|^{n+1}} d \widetilde{\mu} \lesssim\left\|\nu_{Q}\right\| \lesssim\left\|\chi_{Q} f\right\|_{L^{1}(\widetilde{\mu})},
$$


by standard estimates, using the polynomial growth of $\widetilde{\mu}$.

Gathering the estimates for the three terms on right hand side of (5.21) we obtain

$$
\int\left|\mathcal{R}_{\Phi} \nu_{Q}(x)\right| d \widetilde{\mu} \lesssim\left\|\chi_{Q} f\right\|_{L^{1}(\widetilde{\mu})}
$$

which gives

$$
\widetilde{\mu}\left(\left\{x:\left|\mathcal{R}_{\Phi} \nu(x)\right|>\lambda / 2\right\}\right) \lesssim \frac{\sum_{Q \in \operatorname{Reg}}\left\|\chi_{Q} f\right\|_{L^{1}(\widetilde{\mu})}}{\lambda}=\frac{\|f\|_{L^{1}(\widetilde{\mu})}}{\lambda},
$$

and completes the proof of the boundedness of $\mathcal{R}_{\widetilde{\mu}, \Phi}$ from $L^{1}(\widetilde{\mu})$ to $L^{1, \infty}(\widetilde{\mu})$.

Lemma 5.18. The operator $\mathcal{R}_{\left.\mu\right|_{3 R}, \Phi}$ is bounded in $L^{p}\left(\left.\mu\right|_{3 R}\right)$, for $1<p<\infty$, with its norm depending on $p$.

Proof. We first notice that

$$
\mathcal{R}_{\left.\mu\right|_{3 R}, \Phi} f=\mathcal{R}_{\widetilde{\mu}, \Phi} f+\mathcal{R}_{\left.\mu\right|_{3 R \backslash \cup_{Q} \in \operatorname{Reg} Q}, \Phi} f
$$

Recall now that, by Lemma 5.11, $d \sigma(x)=h(x) d \mu(x)$ on $3 R \backslash \bigcup_{Q \in \operatorname{Reg}} Q$, with $h(x) \approx 1$. Therefore,

$$
\begin{aligned}
\int_{3 R}\left|\mathcal{R}_{\widetilde{\mu}, \Phi} f\right|^{p} d \mu & =\int\left|\mathcal{R}_{\widetilde{\mu}, \Phi} f\right|^{p} d \widetilde{\mu}+\int_{3 R \backslash \bigcup_{Q \in \operatorname{Reg}} Q}\left|\mathcal{R}_{\widetilde{\mu}, \Phi} f\right|^{p} d \mu \\
& \lesssim \int|f|^{p} d \widetilde{\mu}+\int_{3 R \backslash \bigcup_{Q \in \operatorname{Reg}} Q}\left|\mathcal{R}_{\widetilde{\mu}, \Phi} f\right|^{p} d \sigma \\
& \lesssim \int|f|^{p} d \widetilde{\mu}
\end{aligned}
$$

where in the last inequality we used the boundedness of $\mathcal{R}_{\widetilde{\mu}, \Phi}$ from $L^{p}(\widetilde{\mu})$ to $L^{p}(\sigma)$, by Remark 5.16.

Without loss of generality, by Lemma 5.17, we may assume now that $f$ is supported in $3 R \backslash \bigcup_{Q \in \operatorname{Reg}} Q$. Thus, in view of Lemmas 5.14 and 5.15, we have that

$$
\begin{aligned}
& \int_{3 R}\left|\mathcal{R}_{\left.\mu\right|_{3 R \backslash \cup_{Q \in \operatorname{Reg}} Q}, \Phi} f\right|^{p} d \mu=\int\left|\mathcal{R}_{\sigma, \Phi}\left(f h^{-1}\right)\right|^{p} d \widetilde{\mu}+\int_{3 R \backslash \bigcup_{Q \in \operatorname{Reg}} Q}\left|\mathcal{R}_{\sigma, \Phi}\left(f h^{-1}\right)\right|^{p} d \mu \\
& \lesssim \int\left|f h^{-1}\right|^{p} d \sigma+\int\left|\mathcal{R}_{\sigma, \Phi}\left(f h^{-1}\right)\right|^{p} d \sigma \\
& \lesssim \int\left|f h^{-1}\right|^{p} d \sigma \approx \int|f|^{p} d \mu
\end{aligned}
$$

where we repeatedly used that $d \sigma(x)=h(x) d \mu(x)$ on $3 R \backslash \bigcup_{Q \in \operatorname{Reg}} Q$, with $h(x) \approx 1$.

Lemma 5.19. The operator $\mathcal{R}_{\Phi,\left.\mu\right|_{3 R}, *}$ is bounded in $L^{p}\left(\left.\mu\right|_{3 R}\right)$, for $1<p<\infty$, with its norm depending on $p$.

Proof. This follows immediately from Lemma 5.18 in conjunction with Lemma 5.5 for $s=1$ and Lemma 5.6. 
Lemma 5.20. Let $R \in$ Top and $x \in R$. Then

$$
\left|K_{R}(f \mu)(x)\right| \lesssim \mathcal{R}_{\Phi, *}\left(\chi_{3 R} f \mu\right)(x)+M_{\mu} f(x) .
$$

Proof. Let us recall that $K_{R}(f \mu)(x)=\sum_{Q \in \operatorname{Tree}(R)} \mathcal{R}_{Q}(f \mu)(x)$. Notice that if $x \in R$ then either $x \in Q$ for some $Q \in \operatorname{Stop}(R)$ or $x \in R \backslash \bigcup_{Q \in \operatorname{Stop}(R)} Q$.

Case (i): Let $x \in Q$ for some $Q \in \operatorname{Stop}(R)$. Then

$$
\begin{aligned}
\left|K_{R}(f \mu)(x)\right| & =\left|\int_{\max (\ell(Q) / 2, \eta)<|x-y| \leq \ell(R)} \chi_{3 R}(y) f(y) K(x-y) d \mu(y)\right| \\
& \leq\left|\mathcal{R}_{\max (\ell(Q) / 2, \eta)}\left(\chi_{3 R} f \mu\right)(x)\right|+\left|\mathcal{R}_{\ell(R)}\left(\chi_{3 R} f \mu\right)(x)\right| \\
& \lesssim\left|\mathcal{R}_{\max (\ell(Q) / 2, \eta)}\left(\chi_{3 R} f \mu\right)(x)\right|+M_{\mu} f(x) .
\end{aligned}
$$

By the definition of $\Phi, \Phi(x) \leq \ell(Q)$, and so by Lemma 5.3,

$$
\begin{aligned}
\left|K_{R}(f \mu)(x)\right| & \lesssim\left|\mathcal{R}_{\Phi, \max (\ell(Q) / 2, \eta)}\left(\chi_{3 R} f \mu\right)(x)\right|+M_{\mu} f(x) \\
& \lesssim\left|\mathcal{R}_{\Phi, *}\left(\chi_{3 R} \mu\right)(x)\right|+M_{\mu} f(x) .
\end{aligned}
$$

Case (ii): Let $x \in R \backslash \bigcup_{Q \in \operatorname{Stop}(R)} Q$. Then every cube $P \subset R$ such that $x \in P$ is in $\operatorname{Tree}(R)$. Thus, it is clear that

$$
\left|K_{R}(f \mu)(x)\right|=\left|\int_{\eta<|x-y| \leq \ell(R)} \chi_{3 R}(y) f(y) K(x-y) d \mu(y)\right| .
$$

Arguing as in the previous case and using that $\Phi(x)=0<\ell(P)$, for every $P \in \mathcal{D}(R)$ such that $x \in P$ (since it is in $\operatorname{Tree}(R)$ ), we can prove (5.22). This concludes our lemma.

Proof of Lemma 5.8. This is an immediate consequence of Lemma 5.20, since both $\mathcal{R}_{\Phi,\left.\mu\right|_{3 R}, *}$ and $M_{\mu}$ are bounded in $L^{2}(\mu)$.

5.5. The boundedness of $\mathcal{R}_{\mu}$ in $L^{2}(\mu)$. In this subsection we conclude the proof of Proposition 5.1 and hence of the implications (b) $\Rightarrow$ (a) and (c) $\Rightarrow$ (a) in Theorem 1.1 and of Theorem 1.3 by showing the following:

Lemma 5.21. The operator $\mathcal{R}_{\mu}$ is bounded in $L^{2}(\mu)$.

Proof. We will argue very similarly to Semmes in [Se]. For completeness we will show the details.

By standard Calderón-Zygmund theory, it is enough to prove that for any cube $Q_{0} \in \mathcal{D}_{\mu}$ and any function $f$ supported on $Q_{0}$,

$$
\int_{Q_{0}}\left|\mathcal{R}_{\mu, \eta} f\right| d \mu \lesssim\left(\int|f|^{2} d \mu\right)^{1 / 2} \mu\left(Q_{0}\right)^{1 / 2}
$$

We consider the corona type decomposition and the family Top given by Proposition 5.1, and then we write

$$
\int_{Q_{0}}\left|\mathcal{R}_{\mu, \eta} f\right| d \mu \leq \sum_{R \in \mathrm{Top}: R \subset Q_{0}} \int_{Q_{0}}\left|K_{R}(f \mu)\right| d \mu+\sum_{R \in \mathrm{Top}: R \not \subset Q_{0}} \int_{Q_{0}}\left|K_{R}(f \mu)\right| d \mu
$$


uniformly on $\eta>0$. It is immediate to check that any summand in the last sum vanishes unless there exists $S \in \operatorname{Tree}(R)$ with $S \supset Q_{0}$ and $\ell(S) \approx Q_{0}$. So this sum has a bounded number of nonzero terms and by Lemma 5.8 we get

$$
\sum_{R \in \text { Top: } R \not \subset Q_{0}} \int_{Q_{0}}\left|K_{R}(f \mu)\right| d \mu \lesssim\|f\|_{L^{2}(\mu)} \mu\left(Q_{0}\right)^{1 / 2} .
$$

To deal with the first sum on the right hand side of (5.23) we use again Lemma 5.8, with $p=3 / 2$, and we take into account that $K_{R}(f \mu)=K_{R}\left(\chi_{3 R} f \mu\right)$ :

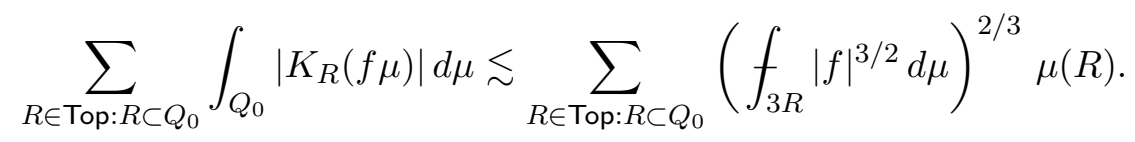

Then, by the packing condition in Lemma 3.9 and Carleson's embedding theorem (see Theorem 5.8 in [To3], for example):

$$
\sum_{R \in \text { Top: } R \subset Q_{0}} \int_{Q_{0}}\left|K_{R}(f \mu)\right| d \mu \lesssim \int_{Q_{0}}\left(\sup _{R \ni x} f_{3 R}|f|^{3 / 2} d \mu\right)^{2 / 3} d \mu(x)
$$

Since the maximal operator

$$
\widetilde{M}_{\mu} f(x):=\sup _{Q \in \mathcal{D}_{\mu}: Q \ni x}\left(f_{3 Q}|f|^{3 / 2} d \mu\right)^{2 / 3}
$$

is bounded in $L^{2}(\mu)$, we obtain

$$
\sum_{R \in \text { Top:R }: Q_{0}} \int_{Q_{0}}\left|K_{R}(f \mu)\right| d \mu \lesssim \int_{Q_{0}} \widetilde{M}_{\mu} f d \mu \leq\left(\int|f|^{2} d \mu\right)^{1 / 2} \mu\left(Q_{0}\right)^{1 / 2},
$$

as wished.

\section{THE PROOF OF COROLLARY 1.2}

We need the following auxiliary result.

Lemma 6.1. Let $\Omega \subset \mathbb{R}^{n+1}, n \geq 2$, be a domain with $n$-AD-regular boundary. Let $u$ be a non-negative, bounded, harmonic function in $\Omega$, vanishing at $\infty$, and let $B$ be a ball centered at $\partial \Omega$. Suppose that $u$ vanishes continuously in $\partial \Omega \backslash \frac{11}{10} B$. Then, there is a constant $\alpha>0$ such that

$$
u(x) \lesssim \frac{r(B)^{n-1+\alpha}}{(r(B)+\operatorname{dist}(x, B))^{n-1+\alpha}}\|u\|_{L^{\infty}(\Omega)} .
$$

Both $\alpha$ and the constant implicit in the above estimate depend only on $n$ the AD-regularity constant of $\partial \Omega$.

Although this result is probably quite well known we will show the details of the proof.

Proof. Assume that $\Omega$ is not bounded (the arguments when $\Omega$ is bounded are analogous). Further, by translating and dilating $\Omega$ if necessary we may assume that $B=B(0,1)$. Denote by $T$ the involution

$$
T(x)=\frac{x}{|x|^{2}}
$$


and consider the Kelvin transform

$$
\widetilde{u}(x)=\frac{1}{|x|^{n-1}} u(T(x)) .
$$

This function is harmonic, continuous and bounded in $B \cap T(\Omega)$. Also, it vanishes in $B \cap \partial(T(\Omega))$ and it is bounded by $C\|u\|_{L^{\infty}(\Omega)}$ in $\partial(B \cap T(\Omega))$, and thus, by the maximum principle,

$$
\|\widetilde{u}\|_{L^{\infty}(B \cap T(\Omega))} \leq C\|u\|_{L^{\infty}(\Omega)} .
$$

It is easy to check that $T$ transforms $n$-AD-regular sets into $n$-AD-regular sets. Thus, $T(\Omega)$ satisfies the CDC, and so $\widetilde{u}$ is Hölder continuous in $\frac{1}{2} B \cap \partial(T(\Omega))$, and for some $\alpha>0$ it satisfies

$$
\widetilde{u}(x) \lesssim \operatorname{dist}(x, \partial(T(\Omega)))^{\alpha}\|\widetilde{u}\|_{L^{\infty}(B \cap T(\Omega))} \lesssim|x|^{\alpha}\|u\|_{L^{\infty}(\Omega)} \quad \text { for all } x \in \frac{1}{2} B \cap T(\Omega),
$$

since $0 \in \partial(T(\Omega))$. This is equivalent to saying that

$$
u(x) \lesssim \frac{1}{|x|^{n-1+\alpha}}\|u\|_{L^{\infty}(\Omega)} \quad \text { for all } x \in \Omega \backslash 2 B,
$$

which proves the lemma.

Proof of Corollary 1.2. We will show that if $\Omega$ is a corkscrew domain with $n$-AD-regular boundary and there exists some constant $C>0$ such that

$$
\|S u\|_{L^{p}(\mu)} \leq C\left\|N_{*} u\right\|_{L^{p}(\mu)} \quad \text { for any function } u \in C_{0}(\bar{\Omega}) \text {, harmonic in } \Omega,
$$

then the assumption (c) in Theorem 1.1 holds for functions that, besides being bounded and harmonic, belong to $C_{0}(\bar{\Omega})$. By Remark 3.8 this is enough to prove the Key Lemma 3.7 and thus the uniform rectifiability of $\partial \Omega$, since the assumption (c) is not used elsewhere in the proof of the implication (c) $\Rightarrow$ (a) of Theorem 1.1.

So we have to show that there exists some $C>0$ such that if $u \in C_{0}(\bar{\Omega})$ is harmonic in $\Omega$ and $B$ is a ball centered at $\partial \Omega$, then

$$
\int_{B}|\nabla u(x)|^{2} \operatorname{dist}(x, \partial \Omega) d x \leq C\|u\|_{L^{\infty}(\Omega)}^{2} r(B)^{n} .
$$

To prove this, let $u \in C_{0}(\bar{\Omega})$ be harmonic in $\Omega$ and consider a continuous nonnegative function $\varphi_{B}$ which equals 1 in $\frac{5}{2} B$ and vanishes in $(3 B)^{c}$, with $\|\varphi\|_{\infty} \leq 1$. Then, write

$$
u(x)=\int \varphi_{B} u d \omega^{x}+\int\left(1-\varphi_{B}\right) u d \omega^{x}=: u_{1}(x)+u_{2}(x) .
$$

Note that $u_{1}$ and $u_{2}$ are harmonic in $\Omega$, continuous in $\bar{\Omega}$, and vanishing at $\infty$, and $\left\|u_{i}\right\|_{L^{\infty}(\Omega)} \leq$ $\|u\|_{L^{\infty}(\Omega)}$ for $i=1,2$.

To deal with the non-local function $u_{2}$ we just take into account that $u_{2}$ vanishes in $\partial \Omega \cap \frac{5}{2} B$ and apply Caccioppoli's inequality. For the application of Caccioppoli's inequality, note that $u_{2}$ is harmonic in $\Omega$, subharmonic in $\frac{5}{2} B$ (when extended by 0 to $\frac{5}{2} B \backslash \bar{\Omega}$ ) and that $u_{2} \in W^{1,2}\left(\frac{5}{2} B\right)$, because it vanishes continuously in $\frac{5}{2} B \cap \partial \Omega$. Then we get

$$
\int_{B}\left|\nabla u_{2}\right|^{2} d x \lesssim \frac{1}{r(2 B)^{2}} \int_{2 B}\left|u_{2}\right|^{2} d x \lesssim\|u\|_{L^{\infty}(\Omega)}^{2} r(B)^{n-1}
$$


which implies that

$$
\int_{B}\left|\nabla u_{2}(x)\right|^{2} \operatorname{dist}(x, \partial \Omega) d x \lesssim\|u\|_{L^{\infty}(\Omega)}^{2} r(B)^{n} .
$$

To prove the analogous estimate for $u_{1}$, first we use Fubini and Hölder's inequality, and then we apply (6.2) to $u_{1}$ :

$$
\begin{aligned}
\int_{B}\left|\nabla u_{1}(x)\right|^{2} \operatorname{dist}(x, \partial \Omega) d x & \lesssim \int_{2 B} \int_{y \in \Gamma(x)}\left|\nabla u_{1}(y)\right|^{2} \operatorname{dist}(y, \partial \Omega)^{1-n} d y d \mu(x) \\
& \lesssim\left\|S u_{1}\right\|_{L^{p}(\mu)}^{2} \mu(B)^{1-\frac{2}{p}} \\
& \lesssim\left\|N_{*} u_{1}\right\|_{L^{p}(\mu)}^{2} r(B)^{n-\frac{2 n}{p}} .
\end{aligned}
$$

From the estimate (6.1) we deduce that

$$
N_{*} u_{1}(x) \lesssim \frac{r(3 B)^{n-1+\alpha}}{(r(3 B)+\operatorname{dist}(x, 3 B))^{n-1+\alpha}}\left\|u_{1}\right\|_{L^{\infty}(\Omega)},
$$

which, in turn, implies that

$$
\begin{aligned}
\left\|N_{*} u_{1}\right\|_{L^{p}(\mu)}^{p} & \lesssim\left\|u_{1}\right\|_{L^{\infty}(\Omega)}^{p} \int\left(\frac{r(3 B)^{n-1+\alpha}}{(r(3 B)+\operatorname{dist}(x, 3 B))^{n-1+\alpha}}\right)^{p} d \mu(x) \\
& \lesssim\left\|u_{1}\right\|_{L^{\infty}(\Omega)}^{p} \mu(B),
\end{aligned}
$$

where we took into account that $n>2$ and so $(n-1+\alpha) p>n$ to estimate the last integral. Therefore,

$$
\int_{B}\left|\nabla u_{1}(x)\right|^{2} \operatorname{dist}(x, \partial \Omega) d x \lesssim\left\|u_{1}\right\|_{L^{\infty}(\Omega)}^{2} r(B)^{n} \leq\|u\|_{L^{\infty}(\Omega)}^{2} r(B)^{n},
$$

and the proof of the corollary is complete.

Note that in the case $n=1$ we can ensure that the integral in (6.4) is bounded by $c \mu(B)$ only if we assume $p>1 / \alpha$. So arguing as above we derive:

Corollary 6.2. Let $\Omega \subset \mathbb{R}^{2}$ be a corkscrew domain with 1 -AD-regular boundary. There exists some constant $\alpha>0$ depending only on the AD-regularity constant of $\partial \Omega$ such that the following holds. Suppose that for some $p>1 / \alpha$ there exists some constant $C_{p}>0$ such that

$$
\|S u\|_{L^{p}(\mu)} \leq C_{p}\left\|N_{*} u\right\|_{L^{p}(\mu)} \quad \text { for any function } u \in C_{0}(\bar{\Omega}) \text { harmonic in } \Omega .
$$

Then $\partial \Omega$ is 1-uniformly rectifiable.

\section{REFERENCES}

[AiH] H. Aikawa and K. Hirata. Doubling conditions for harmonic measure in John domains. Ann. Inst. Fourier (Grenoble) 58 (2008), no. 2, 429-445.

[AG] D.H. Armitage and S.J. Gardiner, Classical potential theory, Springer Monographs in Mathematics, Springer-Verlag London, Ltd., London, 2001.

[AHM ${ }^{3}$ TV] J. Azzam, S. Hofmann, J.M. Martell, S. Mayboroda, M. Mourgoglou, X. Tolsa, and A. Volberg. Rectifiability of harmonic measure. Geom. Funct. Anal. (GAFA), 26(3) (2016), 703-728. 
[AHMNT] J. Azzam, S. Hofmann, J.M. Martell and K. Nyström and T. Toro, A new characterization of chord-arc domains. To appear in J. Eur. Math. Soc. arXiv:1406.2743.

[AMT1] J. Azzam, M. Mourgoglou, and X. Tolsa. Rectifiability of harmonic measure in domains with porous boundaries. Preprint 2015. arXiv: 1505.06088

[AMT2] J. Azzam, M. Mourgoglou and X. Tolsa. Mutual absolute continuity of interior and exterior harmonic measure implies rectifiability. Preprint (2015). To appear in Comm. Pure Appl. Math.

$[\mathrm{BH}] \quad$ S. Bortz and S. Hofmann. Harmonic measure and approximation of uniformly rectifiable sets. To appear in Rev. Mat. Iberoam.

[Dah] B. Dahlberg. Approximation of harmonic functions. Ann. Inst. Fourier (Grenoble) 30 (1980) no $1,97-108$.

[DJ] G. David and D. Jerison, Lipschitz approximation to hypersurfaces, harmonic measure, and singular integrals. Indiana Univ. Math. J. 39 (1990), no. 3, 831-845.

[DS1] G. David and S. Semmes, Singular integrals and rectifiable sets in $\mathbb{R}^{n}$ : Beyond Lipschitz graphs, Astérisque No. 193 (1991).

[DS2] G. David and S. Semmes, Analysis of and on uniformly rectifiable sets, Mathematical Surveys and Monographs, 38. American Mathematical Society, Providence, RI, (1993).

[Gar] J. Garnett. Bounded Analytic Functions. Academic Press, San Diego, 1981.

[G-S] D. Girela-Sarrión, Geometric conditions for the $L^{2}$-boundedness of singular integral operators with odd kernels with respect to measures with polynomial growth in $\mathbb{R}^{d}$. Preprint arXiv:1505.07264 (2015).

[He] L.L. Helms, Potential theory, Universitext, Springer, London, 2014, 2nd ed.

[HL] S. Hofmann and P. Le. BMO solvability and absolute continuity of harmonic measures. Preprint arXiv:1607.00418v1 (2016).

[HM1] S. Hofmann and J.M. Martell, Uniform Rectifiability and Harmonic Measure I: Uniform rectifiability implies Poisson kernels in $L^{p}$, Ann. Sci. École Norm. Sup. 47 (2014), no. 3, 577-654.

[HM2] S. Hofmann and J.M. Martell. Uniform rectifiability and harmonic measure, IV: Ahlfors regularity plus Poisson kernels in $L^{p}$ impies uniform rectifiability. arXiv:1505.06499.

[HMM1] S. Hofmann, J.M. Martell and S. Mayboroda, Uniform rectifiability and harmonic measure III: Riesz transform bounds imply uniform rectifiability of boundaries of 1-sided NTA domains. Int. Math. Res. Not. (2014), no. 10, 2702-2729.

[HMM2] S. Hofmann, J.M. Martell, and S. Mayboroda. Uniform rectifiability, Carleson measure estimates, and approximation of harmonic functions. Duke Math. J. 165 (2016), no. 12, 2331-2389.

[HMU] S. Hofmann, J.M. Martell and I. Uriarte-Tuero. Uniform rectifiability and harmonic measure, II: Poisson kernels in $L^{p}$ imply uniform rectifiability. Duke Math. J. (2014) no. 8, p. 1601-1654.

[JK] D. S. Jerison and C. E. Kenig, Boundary behavior of harmonic functions in nontangentially accessible domains, Adv. in Math. 46 (1982), no. 1, 80-147.

[KKiPT] C. Kenig, B. Kirchheim, J. Pipher and T. Toro. Square functions and the $A_{\infty}$ property of elliptic measures. J. Geom. Anal. (2016) 26:2383-2410.

[KKoPT] C. Kenig, H. Koch, J. Pipher and T. Toro. A new approach to absolute continuity of elliptic measure, with applications to non-symmetric equations. Adv. Math. 153(2) (2000), 231-298.

[Ma] P. Mattila. Geometry of sets and measures in Euclidean spaces, Cambridge Stud. Adv. Math. 44, Cambridge Univ. Press, Cambridge, 1995.

[MT] M. Mourgoglou and X. Tolsa. Harmonic measure and Riesz transform in uniform and general domains. Preprint ArXiv:1509.08386 (2015).

[Pi] J. Pipher. Carleson measures and elliptic boundary value problems. Proceedings, ICM (2014).

[NToV1] F. Nazarov, X. Tolsa and A. Volberg, On the uniform rectifiability of AD-regular measures with bounded Riesz transform operator: the case of codimension 1, Acta Math. 213 (2014), no. 2, 237-321. MR 3286036

[NToV2] F. Nazarov, X. Tolsa and A. Volberg. The Riesz transform Lipschitz harmonic functions. Publ. Mat. 58 (2014), 517-532.

[NTrV] F. Nazarov, S. Treil and A. Volberg. The Tb-theorem on non-homogeneous spaces that proves a conjecture of Vitushkin. CRM preprint No. 519 (2002), pp. 1-84. arXiv:1401.2479

[Se] S. Semmes. Analysis vs. Geometry on a Class of Rectifiable Hypersurfaces in $\mathbb{R}^{n}$. Indiana Univ. Math. J., Vol. 39, no.4 (1990), 1005-1035. 
[To1] X. Tolsa. Bilipschitz maps, analytic capacity, and the Cauchy integral. Ann. of Math.162 (2005), no. 3, 124-1302.

[To2] X. Tolsa. $L^{2}$-boundedness of the Cauchy transform implies $L^{2}$-boundedness of all CalderónZygmund operators associated to odd kernels. Publ. Mat. 48 (2004), no. 2, 445-479.

[To3] X. Tolsa. Analytic capacity, the Cauchy transform, and non-homogeneous Calderón-Zygmund theory, volume 307 of Progress in Mathematics. Birkhäuser Verlag, Basel, 2014.

[Va] N. Varopoulos. A remark on functions of bounded mean oscillation and bounded harmonic functions. Pacific J. Math. 74 (1978), 257-259.

[Vo] A. Volberg, Calderón-Zygmund capacities and operators on nonhomogeneous spaces. CBMS Regional Conf. Ser. in Math. 100, Amer. Math. Soc., Providence, 2003.

John Garnett, Department of Mathematics, 520 Portola Plaza, University of CaliforNIA, Los ANGEles, Los ANGEles, CALIFORnia 90095-1555.

E-mail address: jog@mat.ucla.edu

Mihalis Mourgoglou, Departament de Matemàtiques, Universitat Autònoma de Barcelona, Edifici C FACUltat de Ciències, 08193 Bellaterra (BARCElona), CATAlonia

Current address: BCAM - BASQue Center for ApPlied Mathematics, MaZArRedo, 14 E48009 BILBAO, BASQUE COUNTRY-SPAIN.

E-mail address: mourgoglou@mat.uab.cat

Xavier Tolsa, iCREA, Passeig Llus Companys 2308010 Barcelona, Catalonia, and, Departament de Matemàtiques and BGSMath, Universitat Autònoma de Barcelona, Edifici C FACUltat de CiènCies, 08193 Bellaterra (BARCElona), CATAlonia

E-mail address: xtolsa@mat.uab.cat 